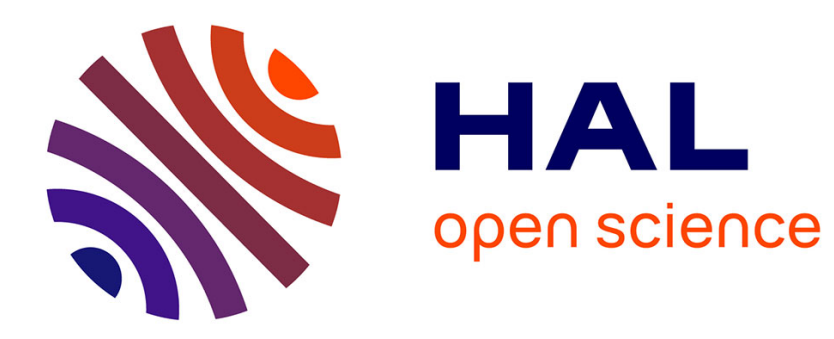

\title{
Why and how to understand what is at stake in a mathematics class
}

Aline Robert, Christophe Hache

\section{To cite this version:}

Aline Robert, Christophe Hache. Why and how to understand what is at stake in a mathematics class. Students' Activities and Teachers' Practices, 2013. hal-01809689

\section{HAL Id: hal-01809689 \\ https://hal.science/hal-01809689}

Submitted on 2 Feb 2022

HAL is a multi-disciplinary open access archive for the deposit and dissemination of scientific research documents, whether they are published or not. The documents may come from teaching and research institutions in France or abroad, or from public or private research centers.
L'archive ouverte pluridisciplinaire HAL, est destinée au dépôt et à la diffusion de documents scientifiques de niveau recherche, publiés ou non, émanant des établissements d'enseignement et de recherche français ou étrangers, des laboratoires publics ou privés. 


\title{
CHAP 2. WHY AND HOW TO UNDERSTAND WHAT IS AT STAKE IN A MATHEMATICS CLASS
}

\begin{abstract}
OVERVIEW
The previous chapter proposed a unique general framework, organized around activities by actors. This framework specifically allows an analysis of student learning and teacher practices. The goal of this chapter is to describe, from a theoretical and methodological point of view, the use of this analysis process to examine the teaching of mathematics in school. All research presented here concerns the teaching of mathematics in middle school and high school (students age 11-18).

Our research has two goals. First, we aim to give researchers access to student learning for a given topic, in relation to the instruction they have received, within a specific school system, from a diagnostic viewpoint (analysis to understand what there is) or a prospective viewpoint (experiments to learn how to enrich the existing situation). Second, we aim in the long term to work on teacher education, particularly based on conclusions from previous analyses and on hypotheses allowed by the theoretical framework (see end of volume).

The goal of this chapter is therefore to describe the specific theoretical frameworks that we use and the general methodologies that follow from these frameworks. Later chapters will describe specific studies led under these frameworks and that use these methodologies.

We will first present the theoretical tools that we have adopted to help us understand the learning of a given topic through examination of the relationship between mathematical content and the teaching and learning of the topic. We will also look at evidence of regularity and variability among classes, teachers, and teaching practices. We will begin by examining how we aim to have access to student learning, and what kind of results we expect to see.

Our approach is, first, thoroughly didactic, in the sense that we develop all our analyses from the specific characteristics of the mathematical content to be taught. This preliminary analysis of content is connected to other in. Thus, we describe the mathematical content which keeping in mind what we will study, student learning. More precisely, in our theoretical framework, mathematical learning is associated with the concept of conceptualization (see previous chapter). This leads us to connect the mathematical content studied to levels of conceptualization. These levels are defined from school curricula and from characteristics of the concepts involved in a set of tasks and in the corresponding knowledge whose use is intended (second paragraph of the first part of this chapter). This is where certain differences can arise between didacticians. For example, we ascribe significant
\end{abstract}


importance, among other characteristics, to the variety of possible ways of making use of knowledge, and we have given ourselves the means to identify this variety from problem statements.

Secondly, for any given topic, we organize our analyses through the study of students' activities. Following the theory of activity, we postulate that student learning depends directly on student activities, even if these activities are partly inaccessible and differ from one student to another, and even though other elements can intervene in student learning (beginning of the first section of this chapter).

Studying student activities involves analyzing their work on assigned in-class tasks, together with anything added by the teacher during class time. We have conducted a priori analyses of tasks in terms of their potential for calling on mathematical knowledge (third part of the first section of this chapter). These analyses allow us to characterize how students must use their knowledge (based on our study of what takes place during class time). We complete our analysis by developing ways to analyze class periods and possible student activities, incorporating all elements added by the teacher during class that contribute to our model of possible student activities (second part of the second section of this chapter).

However, if these activities are well developed for the majority of the class, then we should also take into account, day by day, everything proposed to students on the topic to be studied. We call this sequence of lessons and exercises on a topic the scenario.

We seek to understand global scenarios for a given topic in terms of the intended student conceptualization. These scenarios can be understood as sequences of lessons and exercises associated with intended applications of the content knowledge. This provides an initial approach to understanding possible student activities and student learning. These global scenarios can be seen as planned "cognitive itineraries" (first part of the second section of this chapter).

All these analyses incorporate some general hypotheses about learning. These hypotheses arise from our adaptation of the combined theories of Piaget and Vygotsky for school mathematics learning, as initiated by Vergnaud (cf. previous chapter). The analysis of proposed tasks involves elements that are assumed to have an influence on activities and therefore on learning. Thus, in terms of skill construction, the variety of what students must use in their work plays just as much of a role as the order in which students complete exercises, or the quantity of exercises completed. In other words, the possible ways to mobilize, combine, and recognize the knowledge to be used in exercises are the main factor in constructing student knowledge (along with processes of assimilation, accommodation, disequilibration and re-equilibration). But this depends not only on proposed tasks and the actions they may provoke, but also on the way in which these tasks are worked on by students (particularly in class in terms of the nature and quality of individual and group investment), as well as on the mediations and assistance provided by the teachers. To analyze in-class events, we also use anything that can influence student activities in terms of teacher practices, whether related to the nature of the 
organized work (autonomous, in groups) or to direct teacher interventions (assistance, identification of student work, use of this work, assessments, etc.).

Finally, for the last fifteen years, we have introduced the idea that teachers' choices, in and out of the classroom, are not solely determined by factors related to student learning. They also depend on numerous external constraints, which can be institutional (tied to curricula and schedules) or social (tied to classes and establishments). They can also be tied to the personality, representations, knowledge, and experience of the teacher. To better understand teachers' practices (which depend on student activities and on which student activities depend) ${ }^{1}$, we complete our analyses based on in-class events, taking into account factors tied to the teaching profession through a didactic and ergonomic double approach (third section of this chapter). We use the word "practices" to refer to all work done by a teacher. Although our analyses are based on in-class work and teachers' activities in relation to intended student activities, we include in a teacher's "practice" all work done by that teacher, whether before, during, or after class time.

It is clear that the choice of divisions and variables to analyze is delicate, as the variables in play can be both local and global (a concept or specific exercises, for example). Variables can also be defined in terms of other variables. For example, the way we choose to describe a concept depends on what we find useful in understanding scenarios and their potential for student learning. Descriptions of scenarios, conversely, depend on the specifics of the content involved. Our descriptions of mathematical content (in terms of variety of tasks, for example) should allow for understanding of the corresponding learning process. Finally, if students' intended activities contribute to decoding teachers' in-class activities, it is these in-class activities that in turn allow us to describe possible student activities.

The remaining questions focus on this division, which we will discuss further in the fourth section of this chapter. One open question we continue to work on, for example, is the determination of significant indicators in a teacher's speech. How far should our analysis of the way a teacher addresses students go beyond examining the strict content of the message (which should also be investigated)? To what extent, and to what, are students sensitive: to repetitions, images, spoken and written information, questions, differences in presentations, etc.?

The results that these tools have allowed us to produce, as subsequent chapters will illustrate, come out of relatively recent research, in which researchers have adapted them to particular research areas by specifying, discussing, and enriching the methodology.

This work is equally applicable to the analysis of teachers' manuals, which mainly relies on a priori analyses of tasks and scenarios. This analysis reveals the benefits and limits of the analysis of practices from which we can infer general important characteristics, as mush for students as for trainings: intrapersonal stability, inter-teacher commonalities, variability and changes, etc. A certain number of these studies examine the integration of technology into teaching, and propose new diagnostics of teacher and student difficulties in order to design suggestions for technology use or trainings. 
The reasoned descriptions we produce allow for deep understanding of what takes place in a mathematics class, over the short and medium term, in terms of consistency or diversity for a topic, for a single teacher or multiple teachers. Understanding student knowledge in the long term is difficult, as it is difficult to define and broad, and the variables contributing to this knowledge for each student are out of reach. Thus, even though some studies may relate the instruction given on a chapter, analyzed according to our criteria, to the resulting student work, they are identifying fairly local regularities that depend in part on the individuals involved, and do not claim to deduce from this prescriptive indications. We can say, by contrast, that our research can help to enrich teachers' work, by revealing variables that contribute to their choices before and during class, and by giving them ways to discover the full range of what is possible. In addition, interpreting the identified inter-teacher commonalities and variances within the chosen theoretical framework contributes to reflection on teacher trainings (conclusion of this volume).

In the first section of this chapter we will focus on student activities (intended activities, possible activities, a minima or a maxima activities, etc.). We will present a general outline of our didactic approach, and describe our mathematical $a$ priori analyses of class periods, which will allow us to better understand student activities. These analyses consist of global analyses of the form and type of mathematical content to be taught, and local a priori analyses of assigned tasks in terms of both the intended conceptualizations to be formed of the content and student learning. In the second section, we will describe the a posteriori analyses of class periods, both globally (the scenarios) and locally (analyses of in-class events). These analyses allow us to discover students' activities by using teachers' activities as an intermediary. In the third section, we will detail the analyses of the teaching practices of mathematics teachers, which form the heart of the studies in this volume. Finally, in the two final sections we will discuss the general elements of the methodology, and conclude by indicating methods for comparing different "paradigms" of didactic research.

\section{STUDENT ACTIVITIES AND A PRIORI ANALYSES OF CLASS PERIODS}

\section{Student activities and the general plan for our analyses}

Student activities ${ }^{2}$ (as well as teacher activities) consist of their actions during the completion of a task. This task can be anything from an exercise to listening to a lesson. The activity takes place within a specific situation, such as in class or at home, and consists of external mathematical actions, which may be spoken, written, or performed, as well as internal actions such as hypotheses and decisions as to what to do. These last constitute the student's "personal state." Personal state activity is not directly observable but leaves observable traces. Activities are made up of everything students do, including listening, as well as everything surrounding the actions. This allows the development of knowledge from actions. Student 
activities also consist of what students say, think, do not do, do not say, etc. They depend on a number of factors, including the teacher's activity, which contributes to the desired transformations in terms of knowledge. From the teacher's activity, we use lessons ${ }^{3}$ and assigned exercises as well as work conditions in class and aspects of the teacher's speech. We present in Appendix 2 an overview of the dimensions we believe affect learning. However, there are other factors affecting learning that we do not directly consider, including emotions tied to school, sociocultural factors that can act as a filter between the student and the school, ${ }^{4}$ and factors connected to other circumstances outside of school. These other factors (emotional, socio-cultural, tied to circumstances, etc.) are considered as variable parameter and are taken into account, but are not independently analyzed.

We also do not consider extreme cases of students who do not participate at all in the class activities, whether because they refuse or because they do not understand the transformation expected for knowledge activities. These last students act in ways that are too different from the ways intended. By contrast, some of the studies presented introduce the concepts of action logic (success logic) or learning logic, according to the possible ways to include students in their own learning. The double regulation system from the previous chapter is only used in certain studies, most notably those that explicitly concern individual subjects.

It must be emphasized that students in the same class will not develop the same activities or follow the same course along the same "cognitive itinerary" (see above). An individual's activity also depends on the individual. Elements of differentiation may be introduced in the various studies presented.

As activities are, by definition, partly internal and inaccessible, depending on the case we will only study possible student activities. These activities are presumably close to students' effective activities, but, in light of a priori analyses, we cannot be sure that all students will complete them. We can even, in some cases, be sure that this is not true. When necessary, we identify ways to better approximate students' effective activities (based on computer logs, for example).

From tasks to activities: Possible, a minima, and a maxima activities

All teacher and student actions modify the possible activities, as predicted by the $a$ priori analyses, and contribute to their reconstitution. Among the possible activities, we often distinguish a maxima and a minima activities. A maxima activities are the activities of students who beginning working as soon as the teacher asks. They engage in the assigned task with some autonomy. They often have an idea on how to begin, and are able to overcome the desired adaptations. $A$ minima activities are the activities of students who may be more distracted or slower. They wait until the last moment to begin, and until the teacher has given as many indications as possible. They work with less autonomy. Using a computer, we can more easily identify shifts from the predicted possible activities towards reduced and modified activities.

In each case, we try to identify the reductions, modifications, or enrichments of the activities with respect to the activities predicted by the a priori task analysis. 


\section{Differentiation among students}

By considering real students, even if we do not always examine individual students, we come quickly to questions of differentiation. Several aspects of this differentiation can be investigated. The heterogeneity of classes depends both on differences between students, and of the composition of the class.

Our tools allow us to look at the first aspect from the viewpoints of student results and of teachers' reaction to these differences. This latter includes all the adjustments improvised by teachers, particularly while presenting the correct answer or providing constructive assistance (see further on), which can give us information regarding this differential consideration of students.

Students from the Zone of Educational Priority $(\mathrm{ZEP})^{5}$ have overall representations and general conceptions of school when they enter class. They may be in an environment where school is an unknown or undervalued institution. Parents in the ZEP may be disappointed by school, and young people may expect little from it. Students may be confronted by new external demands that are apparently independent of scholastic acquisitions. This can have consequences on the way they see mathematics. Effectively, if their relationship to knowledge is always an "action" (perform calculations, solve exercises, work on assigned tasks; see Charlot B., Bautier E., Rochex J. Y., 1992) this does not prepare them for the necessity of seeing concepts as mathematical objects.

In class, under various conditions, students begin working on tasks and developing activities. To have positive effects on the conduct of the class as well as student learning, these tasks must be calibrated precisely to be neither too simple nor too complex. Students must move beyond the initial oral solving phase to written work and then to finding links to the knowledge goal. There are many sources of active or passive resistance for students that can be difficult to overcome.

We also observe student micro-actions that are tied to classwork in terms of overall knowledge. Not taking off a coat may indicate that the student has not fully transitioned to class time. Other such micro-actions include not listening from the start, not paying attention (or only rarely), chatting with other students, doing nothing, etc. These micro-actions can also be a permanent obstacle to engaging in the mathematical activity of the class. In particular, a short attention span can prevent students from retaining class events, an act that requires making connections and waiting until something more general emerges. At the same time, these students may be very curious and lively at times, and want to talk frequently, answer quickly, and monopolize teachers' attention. This type of attitude may be harmful in mathematics due to the cumulative nature of knowledge, and the aim of conceptualization.

\section{A schematic for the world of the classroom}

This system is a tentative method for illustrating the subdividing of the world of the study. It is very general and will be used in different ways in each study.

Possible student activities are at the center of this schematic, between teaching practices (upper left) and learning (lower right). The arrows do not have a 
theoretical status, but indicate links that seem to us to be important in our subdivision. If a link is not explicitly taken into account in our research, we represent it with a white arrow. If a link is taken into account, or even drives our research, it is represented by a black arrow. The objects of our research are highlighted in light gray. We highlight in dark gray elements that are observed and analyzed.

Our analyses are narrowly focused on mathematics in school situations, and attempt to take into account the relationships between the individuals involved. However, as shown in our schematic, we focus primarily on the "hands-on" teacher variables. These variables are weighted by factors beyond the classroom, which we do not have the means to completely account for.

As discussed above, our analysis of possible student activities aims primarily to estimate their learning. Our analysis of teacher practices aims to examine ${ }^{6}$ their effects on student activities. Although we do not directly consider external factors on students, we do include some external elements in our analysis of teachers. By taking into account important institutional, social, and personal factors that affect practices, we can better weight, understand, and interpret what takes place.

However, while we can partially reconstruct elements of representation or an overall path, some elements necessarily remain inaccessibly, particularly personal elements. Specifically, for teachers we favor elements of the analyzed situation, such as the mathematics class and the students. These elements, consciously or preconsciously, depend on actors. ${ }^{7}$ While some indicators, particularly in some speech analyses, reveal phenomena specific to individuals, they only reinforce other analyses regarding teachers' choices. These choices primarily concern the mathematical content to be taught and class management. They may be a priori and/or partially improvised, but they are not unconscious.

Global analyses of the mathematics taught and learned in a classroom: levels of conceptualization, types of concepts, relief map

Overall, the way we choose to characterize the mathematical content to be taught should be able to serve as a reference to our analyses of teaching and learning.

We describe students" "acquisitions" in our analyses in terms of level of conceptualization. Following, Vergnaud, we define acquisitions with reference to a set of tasks whose intended resolution requires the reorganization of new knowledge into previously learned concepts, as well as the availability ${ }^{8}$ of a certain number of aspects of the concept. These aspects include objects (definitions, theorems, properties) and tools (contextualized, within different frameworks and registers). The definitions of "tools," "objects," "frameworks," and "registers," which we adapt from those of Regina Douady, can be found in Appendix 1. We also include the definition of "viewpoint."

This starting point leads us to define a level of mathematical conceptualization to be acquired from a given curriculum and for a given concept, domain, or chapter, and contributes to defining the goals of teaching and learning (first section below). However, in order to describe the learning scenarios, we need to add other 
elements to this initial description of mathematics to be taught. These elements affect the choice of the cognitive itinerary to propose to students, particularly tied to the relative proximity of the new concepts to previous concepts (second section). We conclude by introducing the idea of the relief map, connected to all these aspects of concepts that we can take into account before describing a specific scenario (third section).

\section{Relationship with the didactic transposition}

We investigate the didactic transposition between theoretical knowledge and knowledge to be taught by giving ways to define what, in a given piece of knowledge, is prescribed to the study at a given moment. A historical or epistemological study is often necessary to understand what characterizes a concept, the reasons for its emergence at such a moment in history, what of it remains in a given curriculum, etc. However, as didacticians, we often use previously synthesized texts and secondary sources in our research, and do not attempt to advance epistemological research, for example, even if we re-open some questions (cf. Dorier, Bridoux references ?????).

\section{Level of conceptualization}

For us, a level of conceptualization is a fairly large and coherent domain of mathematical work that is at least partly taught (or to be taught). It consists of:

- Fundamental axioms, either specific to the domain or borrowed from other mathematical fields. These may remain implicit at certain levels.

- A corpus of definitions (objects), theorems, and propositions. We call this corpus the level's "arsenal."

- Reasoning methods, steps, and a specific degree of rigor.

- A set of problems that can be resolved within this level.

Within a given level of conceptualization, work may take place in several different frameworks or registers. For example, the geometrical frameworks of points, vectors, numbers, analysis, or figures can all coexist, as can the different registers of Cartesian, polar or barycentric coordinates, various vector notations, complex numbers written in algebraic, trigonometric, or geometric forms, etc. The systems of representations (most notably registers) presented in the previous chapter are again in play here, with the ability to choose such a system and to pass from one to another as an important issue.

The coherence of a level of conceptualization refers to the possibility of establishing a domain's arsenal using only the fundamental axioms and initial definitions. In other words, the domain's theorems can be proved internally, using the domain's tools. This also applies to the field of problems attempted once the arsenal has been acquired. This should not be taken to mean that this should be done with students, nor that there are not other, external, methods to achieve the same results. 
An example

Two levels of conceptualization underlie the geometry taught from middle school to the first years of university mathematics: Euclidean geometry (taught primarily in middle school), and affine and affine-Euclidean geometry (taught in the first years of university mathematics and in preparation for the teaching examination, and introduced surreptitiously in high school). ${ }^{9}$

In "Euclidean geometry," the fundamentals, the work following these fundamentals, and the methods of reasoning all come from Euclid. However, real numbers and area formulas are also included.

We note that these levels do not overlap, even if the body of problems that they can be used to solve may be partially shared. A certain amount of additional generality is acquired in "affine and affine-Euclidean geometry." In addition, there is no strict chronology of these levels in school, with occasional borrowings from a level of conceptualization that has not yet been presented (particularly analytic geometry beginning in middle school, juxtaposed with Euclidean geometry).

The Erlangen program provides another level of conceptualization in geometry that we will not discuss here. The axiomatic geometry developed by Hilbert seems to us to be another candidate for our categorization, and thus a fourth example of a level of conceptualization). We will not describe these two levels of conceptualization, as instructional content is not organized on those bases.

Levels of conceptualization are not simply extensions of one another. ${ }^{10} \mathrm{~A}$ different level of conceptualization is another way of organizing knowledge. Depending on the case, it may represent a generalization (from affine and affineEuclidean geometry to the Erlangen program, for example), or a different focus on the fundamental axioms (from Euclidean geometry to Hilbert geometry), or a change of fundamental axioms (from Euclidean geometry to affine and affineEuclidean geometry).

\section{Relationships with conceptual fields}

Vergnaud's (1990) conceptual fields are defined in terms of students mathematical learning. It is up to the author, as noted in the previous chapter, to provide a framework that "allows for the understanding of the connections and breaks in learning in children and adolescents." The levels of conceptualization that we introduce are much more modest. They are only tied to mathematical knowledge, as developed throughout history and presented in school curricula.

The common use of the word "conceptualization," however, indicates a shared preoccupation with mathematical learning. In our case, these levels organize the mathematical knowledge to be transmitted and contribute to characterizing the expected work at each level, in relation to the variables associated with learning. The theory of conceptual fields enables the conception of a cognitive organization that students should attain for a given conceptual field and an appreciation of the corresponding itinerary. 
Types of concepts

The goal, in this analysis of concepts to be taught, is to understand the relationship between new concepts and concepts students have worked with previously. In particular, we will attempt to deduce the characteristics displayed (and clearly taken into account in school curricula) of reasonable methods of introducing these concepts. One concept may have several introductions, particularly according to the progression previously adopted by the teacher.

We have identified three general types of concepts: Extensions of ideas (with or without "crashes"), RAP concepts (responses to a problem), and FUG concepts (formalizing, unifying, and generalizing). We will discuss their respective introductions later on.

In a mathematics curriculum, "new" can include new concepts (trigonometry in 8th grade, for example) but also new frameworks (the graphical or algebraic frameworks in middle school), new objects (scalar product, introduced in 11th grade), or new theorems and properties (Thales' intercept theorem or the Pythagorean theorem in middle school). We focus on three characteristics that distinguish new concepts (or objects, or frameworks, etc.) from old concepts, and that lead to specific student work. We also analyze the function that these new concepts fulfill in the mathematical landscape where they are introduced. We define these concepts through the combination of multiple such characteristics. Our hypothesis is that each type of concept presented can be introduced in a specific and adapted way.

The generalizing characteristic appears when the new concept is broader than the one students currently have available. The new concept extends the old one, including it to various degrees. It may extend the domain of application, or introduce generality where there was specificity. For example, the scalar product in space generalizes the planar scalar product. Functions can also have this characteristic at the beginning of high school, as students progress from specific, affine functions, defined by their algebraic expression, to general functions, defined as a series of calculations that may not be explicit.

The formalizing characteristic is found in the introduction of a new formalism. This new formalism may be more or less "invasive," and is occasionally used in a limited fashion before its official introduction. New vocabulary (formulations) and symbols may occur in the formalism. For example, the formalism of the framework of elementary algebra is new, particularly due to the appearance of $x$. However, it also contains previously acquired symbols such as $=,+$, etc. and written numbers. These symbols are not always used in the same way in algebra and in elementary arithmetic. The equality sign, for example, represents in algebra not only a result but also equivalence. This is a "crash." As another example, integrals may be introduced to formalize the calculation of the area under a curve. (Integrals also have a unifying characteristic, even if it is not always displayed.)

We highlight, however, that some concepts may have multiple coexisting formalizations, which may or may not fall under different frameworks. This can be seen occasionally when the same name is given to objects. The relationships between these formalisms are not always explicit. The organization of knowledge 
and their representations may be hidden. Authors such as Duval $(1995,1996)$ have done substantial work on the non-congruent correspondences between different registers (writings). He suggests the effectiveness of explaining this aspect of the formalism, assumed to be non-transparent for students.

Examples:

- Cosine (trigonometry in right triangles, scalar product, function);

- $\pi$ (formula for the area of a circle, formula for the perimeter of a circle, complex exponential);

- $\quad$ exponents (arithmetic, with base $e$ );

- linear functions in the plane in the geometric sense (vectors)

- Various theorems

There are also concepts that, even if they are sometimes used implicitly, are not yet formalized or formalizable at a school level (Robert \& Pouyanne, 2004). Arsac (1998) gives the striking example of the distinctions between what we have the right to read and say on a geometric figure (tied to concepts of convexity that are implicit in middle school), what we have the right to read without even saying (tied to concepts of area), and what must be said. Chevallard's proto-mathematical and para-mathematical concepts are of the same kind.

The unifying characteristic indicates that the new concept regroups, brings together, or replaces several elements that were previously treated separately. This unification is often accompanied by a simplification, but potentially also by a loss of clarity relative to the elements that were replaced. Algebraic expressions, for example, when introduced in the new framework of elementary algebra, have a unifying characteristic. The symbol $x$ can equally designate a variable (when statements have an implicit "for any $x$ ), an unknown (when statements are only true for certain values of $x$ ), a parameter, a generalized number that may be an integer, decimal, fraction, etc. Functions also have this unifying characteristic. A function cannot be reduced to its algebraic formula or to its graphical representation, and point, global, and local examinations are necessary to characterize it.

The vector spaces introduced at the beginning of college allow polynomial, series, or vector spaces to be treated in the same way.

We characterize an initial type of concept: some concepts (objects, theorems, etc.) are extensions of older concepts. This may be because they have a generalizing characteristic, or because they are expressed with a formalism that extends a previous formalism. There are "crash-less" extensions, for which old and new work is congruent, and "crashing" extensions, which involve a change in the type of work. Multiplication of decimals, for example, is an extension of integer multiplication. There are no crashes in meaning between the two types of multiplication, but there is a difference in the solving algorithm. The scalar product in space is a crash-less extension of the planar scalar product.

A second type of concept corresponds to concepts that are viewed more as objects, and that are introduced to answer a problem. The problem may be formulated in terms that are accessible to students, and students may be able to begin a solution to it. These types of concepts have two characteristics, which may be generalizing and unifying or unifying and formalizing (for example). The 
Pythagorean theorem may be introduced as the solution to the problem of finding a general relationship between the lengths of the sides of a right triangle. This theorem unifies various specific situations for which students know how to perform the calculations. This is a concept of the type we call RAP (Response to A Problem). Another example of this type is the integral, when seen as the area under a curve (Robert \& Rogalski, 2004). The barycenter may also be introduced as an RAP.

Finally, some concepts will have all three characteristics at once. We call these the "FUG" concepts. FUG concepts allow additional generality while unifying different pre-existing objects using a new formalism. This new formalism often offers simplifications. Two examples have been developed: series convergence and vector spaces (Dorier, 1997; Robert, 1998).

Relief map - student difficulties and naturalization of knowledge by teachers The "relief map" of one or more concepts to be taught is attached to the set of elements that allow us to define what is useful for the researcher (and teacher educator) to know for analyzing teaching. The map is attached to one or more curricula, and includes the mathematical characterization of these concepts. This leads to defining the intended level of conceptualization at a given moment during instruction, as well as the type of concept. The tool and object are specified, along with their integration in previous curricula and in the assumed prior knowledge of students. Other elements involving students, beyond the conceptual structure of the situation, are also defined as cognitive subjects (cf. previous chapter).

We note here the list of previously reported student difficulties, if possible. For example, from numerous didactic studies in elementary algebra, we can introduce the idea that it is necessary to work specifically on the gap between arithmetic and algebra. The difficulties associated with this gap, such as with the new status of the equals sign or the stress on numerical proofs, are often underestimated (Grugeon).

All this allows us to specify the meaning that concepts can take at a given moment of schooling, as well as their place in the landscape of student knowledge. In addition to accessing the distance between new and old, it allows us to identify potential pressure points for teaching and foreseeable obstacles. It also facilitates our understanding of proposed activities covering the concepts, of the design of introductions to the chapters involved, and of the subsequent mathematical work to organize for students, as well as comments to develop and traps that may arise.

Finally, in terms of practice analysis, this facilitates the necessary research in the naturalization of teacher knowledge. The term "naturalized" knowledge refers to knowledge that has become transparent for professionals, but not for students. This knowledge may involve choices of frameworks or changes of viewpoint, for example. Identifying this knowledge contributes to a better appreciation of student progressions (cf. examples in elementary algebra by Lenfant). The passage from a given right triangle to the use of the equivalent property that two straight lines are perpendicular or that a certain angle is a right angle is an example of a viewpoint change with information loss. The given information is not only translated into other words within the same framework (change of viewpoint), but also we are no 
longer considering the triangle as a whole. We retain only the two sides forming the right angle or the angle that they form (placing us in a geometry with explicit measure).

Using a global view of the relief map on a studied concept facilitates the focus on key elements that may intervene in teaching or learning.

To go further in the analysis of taught mathematics and attempts to give a useful relief map to teaching, it is possible to introduce levels of conceptualization that cover all of formal schooling (Dorier, 1997; Robert, 1998). The concept of conceptual field (Vergnaud, 1990) is another way to access this attempt that is perhaps more adapted to the first degree. Very generally, it is useful to analyze networks of concepts that are studied together (Robert, 1992). The analyses developed by Chevallard (Chevallard 1992), more systematic than those indicated here, allow a very complete approach to taught mathematics, from the starting point of didactic transposition and decision theory.

\section{A priori local analyses of mathematical tasks}

A mathematical task is, here, very generally, attached to a given statement proposed to students. It is characterized by the use of old and new knowledge to solve it. The various ways of using the knowledge are determined according to course content (theorems, definitions, properties, examples, solved exercises, etc.). What interests us here is the way (or ways) in which students can use their knowledge in the exercise. This allows us to predict possible students activities for a given problem statement, particularly in class.

These analyses are called a priori as they may be based only on the problem statement, without examining cases where the problem was solved by one or more students or by a class accompanied by a teacher.

The a priori analysis of a task leads to asking, for a curriculum, what the role is of exercises in in-class work, and what the use is that students will make of their old and new knowledge in working on the problem. This analysis, then, does not refer directly to the potential learning benefits of an exercise. We are only trying to find what activities students will be able to take part in for this exercise, with their supposed knowledge (curricula, previous lesson content, etc.). But even if they do not explicitly appear in analyses, the choices made for describing these activities are certainly not independent of hypotheses concerning learning.

For example, we determine whether or not the knowledge to be used is indicated in the problem statement, and if so, whether this indication is direct or implicit (an implicit indication may be given by the placement of the problem in the lesson progression $)^{11}$. If the knowledge to be used is not indicated, it may be assumed to be readily available for students. This indicates the necessity of a specific and fundamental activity for students to allow them to access this knowledge or think of using it (two activities that are difficult to dissociate). We hypothesize that this activity may contribute to constructing the desired availability. 
We first distinguish simple and isolated tasks (SIT), or immediate applications of a piece of knowledge without adaptation or combination. A single piece of knowledge is used, potentially with simple replacement of general inputs by the given information in the context of the exercise.

\section{Different levels of knowledge use}

When tasks are simple and isolated, we speak of student work at the technical level. When $\operatorname{tasks}^{12}$ require adaptations of knowledge that are at least partly indicated, we speak of the level of knowledge application that can be mobilized. Students' work is not effectively analogous, depending on whether they must look for the knowledge to use (questions of why or what), or apply and adapt the indicated knowledge (questions of how). If it is up to the student to recognize the knowledge to use, we speak of the available level of knowledge application.

Rising to a certain level of knowledge application for a given task requires that the student's work on the task involve the knowledge at this level. Either this is possible for this student, and the knowledge is perhaps reinforced, or it is not initially possible, and working on the problem will perhaps contribute to transforming the student's knowledge until it is possible.

\section{Knowledge adaptations}

For other tasks, we determine, for each relevant piece of knowledge, the adaptations that students must do, in relation to the required recognitions, initiatives, additions, and combinations (Robert, 1998; Robert \& Rogalski, 2002). This allows us to characterize individual problems, each of which may involve multiple tasks. These analyses clearly depend on the given level of schooling or the given class. We also keep track of the set of proposed tasks and their repetitions.

Recall the importance, accepted for mathematical learning, of the variety of contexts encountered, and of their interactions, particularly changes of frameworks, registers, viewpoints, and combinations of old and new.

We have developed a list of seven adaptations. We completed this list by considering activities students may have to perform using raw pieces of knowledge, and distinguishing among them recognition of properties or procedures or procedure application, or what is an introduction of intermediaries or steps, which seems to us to be another very important mathematical activity. We also distinguish combinations, links, or changes among elements such as frameworks, and work further on different types of intellectual activities that are specific to mathematics.

These adaptations (identified with a code of type Ai) may occur simultaneously. Each has a fairly large (and again, relative) spectrum:

- A1. Partial recognitions of ways of applying concepts, theorems, methods, formulas, or other types of knowledge. For geometry, this typically consists of recognizing configurations, using Thales' intercept theorem, etc.). This can range from recognizing variables and notations to recognizing formulas, conditions of applying formulas, etc. 
- A2. Introduction of notations, points, or expressions as intermediaries. In geometry, this typically consists of introducing a parallel line, or naming a point to use Thales' intercept theorem. ${ }^{13}$

- A3. Combinations of several frameworks or concepts, point of view changes, framework or register changes, connections, or interpretations, etc. In geometry, this typically consists of using algebraic calculations for obtaining the result (for example, solving $x 2=1$ within a geometry problem). Problems that involve graphical/algebraic aspects of functions automatically contain this adaptation.

- A4. The introduction of steps, or the organization of calculations or reasoning processes. This can range from the repeated use of the same theorem to reasoning reductio ad absurdum using this theorem. In geometry, this typically consists of using Thales' theorem and its converse four times, nonindependently. The steps can be classical, somewhat forced (in examining a function), or to be determined.

- A5. Use of previous questions in solving a problem.

- A6. Using choices, which may or may not be forced (only one will lead to the correct answer).

- A7. Lack of new knowledge.

Let us examine the following exercise, as an example of a problem given at the end of middle school: "Show that the product of two numbers, each of which can be written as the sum of two squares, can be written in the same way."

An initial activity will be to understand and formalize the given sentence. What is "a number that can be written as the sum of two squares"? How should "can be written in the same way" be interpreted? We can note that the question is open. One step is imposed on students: to know how to form a conjecture on the result to be demonstrated. This may induce numerical experiments, but we can suppose that they will not change the search for a proof. An "elementary" response is: "If $m, n$, $p$, and $q$ are integers, we can write:

$$
\begin{aligned}
& \left(n^{2}+m^{2}\right)\left(p^{2}+q^{2}\right)=n^{2} p^{2}+m^{2} q^{2}+2 n p m q+n^{2} q^{2}+m^{2} p^{2}-2 n q m p \\
& =(n p+m q)^{2}+(n q+m p)^{2}
\end{aligned}
$$

And thanks to the stability of integers under addition and multiplication, we can conclude the desired result."

In addition to elementary algebraic manipulations, it is necessary to introduce an intermediary: the algebraic expression $2 n p m q$ that we add and then subtract. The useful identities are, in this method, knowledge items that must be adapted.

Another possible response uses complex numbers. This represents a change of framework, as the problem statement was arithmetical. Under this method, each sum of squares is identified as the square of the absolute value of a well-chosen complex number (the intermediary). We thus write $n^{2}+m^{2}=|m+\mathrm{in}|^{2}$, and then apply the rule that $|z|^{2}\left|z^{\prime}\right|^{2}=\left|z z^{\prime}\right|^{2}$ (rule adaptation) and invoke the stability of integers under addition and multiplication.

In 12th grade, when students have learned complex numbers, there can therefore be a strategy choice for students. 
ALINE ROBERT, CHRISTOPHE HACHE

In the case of computer-based lessons, new tasks appear in addition to the associated new activities. In all cases, task analysis should take into account the software environment, which can simplify or complicate the proposed tasks and modify the possible student activities. To a lesser extent, the analysis of textbook tasks can also take into account the environment. In that case, it consists of external indications such as titles or images that may or may not help the student's activity.

We note that there are other task analyses that less directly involve the specific knowledge to apply for a given exercise, but instead refer exclusively to the nature of the expected work: conjecture, search for a proof, applying a procedure, etc. (Stein, 1996).

Analyses in terms of competencies focus on the broad types of intellectual activities, defined independently of content (looking for information, beginning on a process, communicating, etc.). These do not refer directly to the knowledge involved, introducing a fundamental difference between competencies and knowledge. However, a competency allows for the possibility of solving tasks that are not simple, that are varied, and that are not "ready-made." This includes reallife issues, and supports the possibility of diverse available knowledge adaptations, which allows for the use of the previous analyses.

Other approaches will be presented in the last section (particularly a decision theory analysis).

\section{A POSTERIORI LESSON ANALYSIS}

\section{Global analyses of scenarios presented to students}

For a given instance of instruction, the "scenario" refers to the intended ordered set of exercises and lessons for a chapter or concept. ${ }^{14}$ It includes evaluations and homework, with rough predictions of management (length, division of work). A scenario is understood both in terms of its "internal" qualities, on which depend the set of activities that we can predict through a priori analyses, and in terms of the actual events it permits, beyond its own content.

We study the overall scenario, first under the predicted method of introduction, relative to the specifics of the concept (particularly its similarity to previous concepts). This is the first introduction of the meaning of the concept. We then examine the scenario's lesson/exercise dynamic and the corresponding dynamics, which can exist between meaning and techniques (cf. what Douady in particular calls familiarization or reinvestment). Finally, the quantity and the nature of the proposed tasks are associated with the evaluation of the adaptation work proposed to students. These elements (various dynamics presented, adaptations under student initiative, etc.) are also elements that allow us to think that the implementation of a scenario would allow a larger availability of the concept, and a certain (re)organization of knowledge. 
This does not completely prejudge what may happen in class. It is the analyses of class events that can provide information of this point. We call a scenario "robust" if it can play out in multiple ways in class without modifying the main element, student activities.

Scenario analyses allow, among other things, shedding light on the double role of the teacher's work:

- Design scenarios that aim at constructing meaning out of what is taught and the necessary technical acquisitions; and

- Organizing classroom events to allow convergence between what was envisioned and what takes place in reality.

We will now discuss the two dimensions mentioned above: the introduction of concepts, and the contextualization/de-contextualization dynamic.

\section{On concept introduction}

The problem is made difficult by the diversity of concepts introduced to students in curricula. In our eyes, the introductions depend on the concepts in play. From this, for us, one important variable involves the identification of the desired concepts (detailed in the second section of this chapter) and its appropriateness for the proposed introduction.

The question of concept introduction seems to us to be addressable thanks to our classification of types of concepts.

A concept deemed an extension may be, for example, introduced through a problem, constructed with the "old" knowledge. The problem will be accessible, since the new concept is an extension, but the solving of the problem will require the new concept.

The problems arising from work on the tool/object dialectic also seem to us to correspond to extension-type concepts, particularly if the extension is without a crash (see section 2.2). These problems allow us to broaden the meaning of the new concept and the associated technique, provoking for students an initial use, as an implicit tool, of what is intended. If there is a crash, we can still introduce the new concept in the same way, but the crash can lead to an error. It is the quality of the problem, particularly in terms of the predicted internal methods of control (if possible) that will allow the obstacle to be surmounted.

The concepts that may correspond to a mathematician's responses to a problem (RAP) may be introduced by giving students a problem that they can appropriate and understand, but not solve. In our classification of adaptations under student control, this corresponds to adaptation A7 (see section III). It is the teacher that will introduce the new concept, particularly the new object with, if necessary, its formalism. While working, students can partially test out to what the new concept brings a response. We understand that these introductions are more relevant to the meaning of concepts than to new techniques (particularly if there is a new formalism as well).

For FUG concepts, we hypothesize that there is no problem adapted to introduce them with meaning. The introduction that we can suggest is very partial. It is possible that the optimal strategy is to present part of the knowledge first (or very 
quickly), and then give students a problem to give it meaning (cf. Dorier et al., 1998, on linear algebra; Robert, 2011, on series convergence). Unless the nature of the tool can emerge from what students already know, without them noticing that they are missing something, this process consists of leading students to work with these concepts. We may ask whether the introduction of "informational jumps" (Brousseau, 1998) does not have something to do with this type of factor. Students must be "forced" to use the elements introduced in the course, even if they are not familiar and do not initially seem efficient to students. In the case of informational jumps, there is the idea of a "forced" efficiency. For us, there is even a contract effect.

The contextualization/de-contextualization dynamics and the variety of proposed adaptations

Students' activities prepare them, to a certain extent, for the intended conceptualization. Inspired by theories of learning and research results in mathematical didactics, we have identified dimensions that may influence the quality of activities proposed to students, in relation to this intended conceptualization, and therefore the intended availability of concepts, tools, and objects. What we call the "meaning" of concepts corresponds to this characteristic of acquisitions. Adding meaning translates (and is translated by) the possibility of applying the concept wisely, in various contexts. In light of our hypotheses, is implies (and is implied by) student work that puts into play, in a dialectic manner, the tool and object characteristics of the concept, tied to the exercises and lessons, and the organization of new concepts in the entire knowledge set, tied to the variety of the proposed tasks. Ideally, this consists of introducing new knowledge within a certain continuity as much as possible. Knowledge should be introduced alongside old information, within a context that allows a particular tool-type use, relative to the intended level of conceptualization. This assures (in part) the possibility of the beginning of an autonomous construction. This implementation also assures the possibility of moving past this, thanks to a teacher that presents decontextualized object characteristics, which may be re-contextualized in other ways due to the introduced generality. We see clearly the importance of choices of tasks and lessons (knowledge presentation).

An initial issue thus relates to the way in which knowledge is introduced, in relation to the exercises. The order of what is applied during the exercises and what is presented in the lesson allows us to understand the connection between what is worked on in context and what is presented out of context.

The tasks proposed to students introduce them to diverse aspects of the concept, and diverse ways to work on it (adaptations). It is in studying the desired implementations of knowledge, and the relative variety of the adaptions, in relation to the specifics of the concept, that we can understand the span of the intended knowledge and the foreseeable reorganization of the new into the old.

We should note that each concept requires a specific analysis. An example of discussion on the scenario is given for instruction of orthogonal symmetry in sixth grade (Chapter 7). 
It should also be said that it is the in-class activities that lead to all these tasks. Their analysis is indispensible for understanding what is in play. These analyses are prefaced by specific a priori analyses of tasks, and analyses of in-class events that are discussed in the following section.

\section{Local a posteriori analyses: in-class events}

A number of factors influence students' in-class activities and, as a result, the knowledge created. These factors contribute to encouraging the transformation of presented information into individual understanding through the intermediary of student activity. There is, however, no general law that connects teaching and learning. We can only note common elements, which depend strongly on work conditions, classes, and the types of knowledge in play. For example, an action, even if repeated, does not necessarily generate a construction of knowledge. There must be a transformation of this action into an activity.

$\mathrm{Be}$ that as it may, it is the analyses of the relationships between the expected tasks and the events organized by a teacher during a class period that allows the researcher to understand what are the possible student activities. It consists of giving ways of answering the following question: to what extent have students performed the activity that was expected from the a priori task analysis? What was the nature of their activity? Recall that in general, we cannot claim to be able to access the effective activities of each student. We can only access their possible activities. However, these local analyses remain partial and should be connected to the set of what is proposed to students.

A number of examined elements contribute to the development of the answer to our question. First, the form and nature of the work are recomposed and divided into episodes (see below). These elements clarify student activity, and lead to inferring its potential non-didactic characteristics, the possible role of inter-student interactions, the importance of the activity during the class period, etc. One important source of observable elements is tied to factors added by the teacher, whether in soliciting student responses, responding to students, or developing a didactic project.

In any event, it is the supplementary analyses that allow us to recompose all the information and suggest a reading of possible student activities in a given class period.

The didactic contract, ${ }^{15}$ along with the habits, customs, and memory of the class, also plays a role in learning. Students may, for example, engage in a task because they have understood that it is expected of them by the teacher, and not for mathematical reasons. What do they learn from this? We also take into account this type of overall interrogation.

We indicate below several dimensions that may guide our analyses. 
Nature and format of student work, including autonomous work, group work, written work, oral work, etc.

In our analyses of class periods, we note, in addition to the duration of student work on the tasks proposed to them, ${ }^{16}$ the format (as a class, in small groups, etc.) and the nature of the in-class work (re-copying, reading, calculation, investigation, written or oral, graded or not, etc.).

This allows us to bring to light, at least in part, the autonomy given to students (including not doing what the teacher expected), the role of exchanges between students, and the possibility or necessity for students to take initiative, whether tied to the intended adaptations or to others. This should naturally be completed by the manner in which this work is "recuperated" by the teacher and related to the supposed state of student knowledge. ${ }^{17}$ To the extent that we hypothesize, following Piaget and Brousseau, the importance (and, indeed, necessity) of individual moments of knowledge construction, we understand the value of occasions where one or more students confront a problem autonomously. We therefore identify occasions where students are left to work on their own, either in the long term (a non-didactic phase) or not. During these phases, the teacher has no influence whatsoever on students, neither by assistance nor by direct or indirect indications. The nature of students' mathematical activity then depends on the object of the work, in relation to their knowledge: preliminary investigation following the introduction of a concept, or solving an exercise in a given chapter, or during a problem that cuts across several domains, etc. There are many parameters to include in analyses during the reconstruction of possible student activities.

From this point of view, class periods involving computers interest us particularly to the extent that autonomous student activities is generally more present a priori (see also Chapter \#\#).

To the extent that we hypothesize that student exchanges, during interactions between students, ${ }^{18}$ represent socio-cognitive conflicts, and that teacher interactions influence student activities, we aim to take this into account. This is particularly true in analyses of the existence and nature of the exchanges (predicted or not by the organization of the work provoked by the teacher), as well as analyses, during group phases, of the verbalizations (formulations, formalizations) requested from students. We will return to this below.

Studies in education sciences, particularly in the framework of socially underprivileged students (reference) has long insisted on the importance and specifics of students' written work (in all disciplines). Written work is an occasion of distancing oneself from action. We hypothesize that this is something that must be addressed in knowledge constructions. In mathematics, in particular, this written work is both a method of representation and an instance of work in formalization or symbolization. There can even be some unexpected creativity in mathematical production based on symbolic writing (drawing a figure or writing a formula on paper can put students on the path to a proof or interesting calculation that was not anticipated). 
In class, the use of written work is fairly variable, as is its relationship with oral work. Very different forms of written work exist (provisional or draft work, for example). We can hypothesize that the effects of this work are not the same for different students. This dimension is still under construction in our research.

The teacher's written work (particularly at the board) is another object of study. We have previously shown the regularity of forms used by each teacher (cf. Vandebrouck, Robert, \& Vandebrouck). One important question involves the role given by teachers to their own written work. Is it a simple translation of what they said aloud? Are there transformations between written and oral work? Are they indicated or implicit? Does the writing on the board or on handouts serve as a model for students' written work? There are many such questions, whose answers can bring light to the corresponding student activities, and particularly what is left under their control (which can be a source of potential misunderstanding for students who do not decode it).

In the case of computer-based classes, this written work, and particularly its articulation with the machine work, becomes a very important variable for student activities (see Chapter \#\# in this volume).

\section{Teacher interventions}

Multiple aspects of teacher intervention were analyzed, always in terms of their supposed influence on student activities. Some relate to the format of interactions with students, and others concern the content of the interventions (assistance, assessment, reminders, explanations, corrections and evaluations, presentation of knowledge, mathematical content, etc.). In the background, we find the attention given to the identification that the teacher makes of students' visible work, and to the possibility of profiting from it, by calibrating interventions to knowledge that is assumed to be "close" to students' level. Everything that contributes to this identification, such as questions, answers, or throwing the initiative back to students, may also be an object of study (see the second part of Chapter 2). Aspects of interactions with students have long been studied by numerous authors, ${ }^{19}$ without considering the content in play (Postic).

One important variable that can affect the importance of interventions relates to the student knowledge to which these interventions relate, and more precisely to their degree of proximity to students' previously acquired knowledge. ${ }^{20}$

\section{Focus on assistance}

We define the nature of teacher assistance, identifying the moment when the assistance what given, the nature of this assistance, and the format. We present two types of assistance according to whether they modify the activities predicted $a$ priori, or whether they add something to students' actions.

The first type, said to have a "procedural function," involve the assigned tasks themselves by strictly modifying activities relative to those predicted from the $a$ priori analysis of the problem statement. They correspond to indications given by the teacher before or during student work, and include open-ended questions such as "What theorem can you use?". They may lead to subdividing the task into 
explicitly mentioned subtasks, or to having students choose a contextualized method. This changes the necessary adaptations, and can orient the activity toward

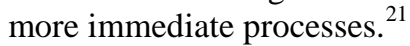

The other type, whose function we call "constructive," add something between the specific student activity and the desired knowledge construction that can form as a result. This may be through a simple summary of what was done, even in an immediate application (for a simple isolated task), or by reminders, partial generalizations, assessments, etc. All kinds of interventions lead students to gain perspective on what they have done, to find a slightly more general method, to discuss results, etc. This assistance can present a small de-contextualization of what students have done, by presenting the corresponding generic case, for example. It can also indicate how to do this type of task, or explain certain choices.

For a given student, procedural assistance can immediately become constructive, if the student extracts a generalization. For a different student, constructivist assistance can remain procedural. If the value of the generality is not understood, the student will forget it once the exercise is over and never use it again.

Recall that adopting the framework of the theory of activity leads is to hypothesize that there is another "internal" transformation of the subject. In solving a problem, the student must live between the solving and the knowledge that will be potentially constructed through this process. This constitutes a depersonalization, a generalization, and finally a de-contextualization and/or an organization. Constructive assistance participates at least at the beginning of this process.

This process is often unsuccessful, especially for students with the most difficulties in the subject. It is one of the most fundamental issues for teachers in Zones of Educational Priority. We think it can also be assisted by developing the knowledge as a group, before assessments. This can lead students to make a place for this knowledge before they have it (Butlen \& Pezard, 2003). This is another form of intervention to facilitate the previous process.

We note also that the way in which teachers consider individual students in group interactions to further their goals for the class is also a factor in the potential influence of assistance. Will the teacher look for information to regulate the interventions, particularly during the presentation of the correct answer? Will the teacher rely on the strongest students? What assessment will the teacher make of the class from individual assessments?

Focus on the quality of the speech (linguistic functions and linguistic markers)

Some finer characteristics of speech contribute to modeling student activities, particularly during their work on complex tasks (Chapter 2, part 2 ???). These finer characteristics include the nature of linguistic functions engaged during interactions, the regular use of certain linguistic markers at certain specific moments, and other characteristics tied to indicators that are yet to be determined. Through this type of speech analysis, we can better understand the way to 
accompany and influence student work. Whether this reinforces teachers' other choices, or compensates for them, is an open question.

In some research, supplementary indicators are used to analyze practices. These indicators relate to teachers' automatic actions, such as simple routine professional gestures (including oral gestures). This analysis leads to defining the speech presented to students more precisely, at finer levels, including aspects of which teachers are not aware.

A focus on correction phases and lessons (phases of knowledge presentation) Phases during which the teacher makes use of student work represent an important variable in student activities, related to the synthesis of their actions.

We differentiate several types of correction phases (oral, written, continuous, at the end of an exercise, by the teacher, by students, etc.). This type of reflection may be compared with reflections on the role of errors in learning.

Hidden behind some errors are false or incomplete representations that may remain in place for students if nothing specific is said on the subject, or if nothing is asked of them during the correction phase to bring them to light. The presentation of the model solution can leave then in silence, unnoticed by the professor or by students.

In addition, multiple elements may come into play during the correction phase. These include the specific solution to the questions, but also what "worked" in the exercise (de-contextualization of the method), how to write it up, etc. ${ }^{22}$ In particular, when the solution to each exercise is presented as students complete it, more general aspects of the exercise may escape students unless their attention is brought to it, overall, at a given moment.

In a way, the correction phase can be a fruitful time for the student's action. It can contribute to the transformation of the student's action into internalized knowledge. One way this can occur is if the student has succeeded at the assigned task and the teacher's confirmation and summary has allowed the student to retain some aspects. Another is if the student did not succeed, but the teacher has responded to the student's attempts and helped complete them, allowing the student to progress. We do not see all correction phases as equivalent. They should both add generality and be close to students' processes. The teacher should remain, if possible, in students' proximal development zone.

Studies on knowledge presentation phases (lessons, institutionalization when this follows certain situational formats) are few.

However, there are several variables noted, and some overall dynamics that have already been mentioned: the order in which the different phases take place, relationships between contextualization and de-contextualization, or even the format of the course (lecture-based, interactive, dialogue-based). The nature of students' activity during the phases of the course is clearly an important variable (even if it is difficult to analyze when their only observable action is listening).

We also examine the nature of the comments on the mathematics (or "meta" comments). We determine whether these types of comments, which refer not to strictly mathematical knowledge but to a larger reflection on this knowledge, or to 
a reasoned presentation of possible methods, exist. These comments may also consist of an explicit external structuring of the lesson and particularly the proofs (argumentations), or recounting the emergence of knowledge in relation to the problems that have appeared, for example in the case of FUG concepts for which no introductory problem is proposed (cf. Dorier). Meta comments may also include a presentation of subsequent occasions to use the concept.

Some studies have attempted to classify methodological comments by their distance from the intended content. This classification distinguishes general comments on work, such as methods that are independent of the specific problem where they are being used, from methods specifically adapted to the problem (cf. Robert \& Tenaud).

A few other studies have focused on teachers' examples, metaphors, or formulation progressions. These studies have examined natural language and symbolic vocabularies (cf. semiotic studies), as well as differences between spoken and written communication. Finally, class handouts and documents remain unstudied.

\section{Work outside of class}

Work outside of class probably becomes more important as students progress through school. There have so far been few studies examining out-of-class work, although much ink has been spilled on the topic (concerning "coursework inflation," for example). It seems coherent with our theoretical framework to assume that homework is not independent of classwork, and may even depend on it (Félix, 2004; Rayou et al., 2010). We do not directly include it in out analyses except when it leaves traces in the classroom: Exercises given to students to complete at home that are then corrected in class, etc.

\section{Open questions}

As we noted above, there are still some open questions in this field. These include the long-term influence and, more generally, the "size" of the topic.

Some studies take into account this important dimension. They may compare, for example, the set of activities on a chapter to students' abilities at the end of instruction. This requires adapting the methodology to handle a considerable quantity of data (cf. chapter 6 and chapter 7 in this volume).

\section{ANALYSES OF THE PRACTICES OF MATHEMATICS TEACHERS (THE DIDACTIC/ERGONOMIC DOUBLE APPROACH)}

This section is developed a bit more than the others, to the extent that it corresponds to more recent research and to an enlargement of previous frameworks. We will present a brief history of the theoretical evolutions before describing the current method of analyzing teacher practices.

We begin by noting that the word "practices" is used to refer to everything that informs us of teachers' thoughts, which may include speech or actions. The viewpoint is long-term, and includes periods before, during, or after class. The 
term "activities" is reserved for specific moments within these practices, and refers to specific situations in a teacher's work: in-class activities, preparation or testwriting activities, cooperative activities, etc. The work "work" is reserved for the subset of practices within a mathematics class and in preparation for this class that constitute the heart of our analyses.

This distinction is important to the extent that we believe that it is necessary to introduce concepts specific to the study of teachers' practices and activities. For example, in some studies, we examine teachers' activities within a specific situation of integrating technology in their classrooms, and adopt an approach that is directly inspired by professional didactics. In these studies, the dialectic between "productive activity" and "constructive activity" is introduced to specify that the teachers act on and transform the situation (contributing to students' activities) but also that teachers transform themselves through a long-term development process.

\section{A brief history: What role to give to teachers in our analyses?}

Many of the researchers contributing to this project work in teacher education (in Ecoles Normales, ${ }^{23}$ and then in Instituts Universitaires de Formation de Maîtres ${ }^{24}$ since 1991). Whether in initial training or continuing education, the difficulty of diffusing research in mathematical didactics is constant. Why is it so difficult? A scientific explanation of this phenomenon seems necessary.

Additionally, like many didacticians in recent years, our research in the links between teaching and learning has led us to focus on effective activities in class, such as student activities provoked by the teacher's speech.

\section{Initial attempts}

Some studies on mathematics teachers were conducted in the 1990s. Starting from the observation that teachers had difficulty listening to didacticians and adopting didactic inventions, we initially wondered if this might not be due to differences between teachers and didacticians in representations of mathematics, of mathematics teaching, and of mathematics learning. It was quickly shown that this explanation was not sufficient to explain the observed differences (Marilier, 1994), and was even less useful for acting. In the first place, the expressed representations to which we had access did not sufficiently translate or explain effective practices. Secondly, teachers' difficulties in "borrowing didactic elements" and the gap between the possible and the (prescribed) didactic could not be ascribed solely to people, but also had to be related to something else, particularly what we will introduce later as the "profession."

In order to explore teaching practices, we focused on in-class speech, both in terms of the mathematical content of the tasks assigned to students, as well as additional content added by the teacher.

For several years, studies have shown that there is inter-individual variability in inclass speech, in meta commentary (Chiocca, 1995; Josse \& Robert, 1993) and contextualization of the same problem. Methodologies were borrowed directly form mathematical didactics. In addition to analyzing tasks given to students in 
terms of the specific intended content, researchers also analyzed speech through a general categorization that distinguished structure, argumentation, nonmathematical accompaniment, etc. Only students were taken into account, and content aimed at them was the only yardstick used by the analyses.

The last study of this type was Hache's 1999 dissertation, which succeeded in regrouping certain variables tied to speech, management, and content, identifying several "universes" unique to each professor from a number of possible types. Each universe was characterized by a certain combination of the nature of the proposed tasks and the corresponding management (characterized by the nature of the elements relative to the teacher's speech). A single teacher never took from more than 3 or 4 universes.

At this stage, there were still large questions remaining beyond the observation of this diversity. These concerned not only the effects of this diversity on learning, but also on the interpretation of this variability, and the consequences we can identify for teacher education. Why do teachers, whether beginning or experienced, use such or such methods to lead a class? What variation exists for a single teacher? Between teachers? At the same time, some studies on teacher education (Masselot, 2000; Vergnes, 2001) have pinpointed beginning teachers' difficulties in borrowing elements from didactics for their practices. It may be tempting to say that we will give students a "good" problem, a problem that will prepare them for constructing the meaning of a concept, but there may nevertheless be obstacles. Instruction may consist only of introductions to concepts. All concepts may not be equally suited to this type of approach. There may be time constraints that affect the possibility of letting students work autonomously. All students may not be equal, including in how prepared they are for autonomous work. Beyond the introduction, the technique must also be practiced. Finally, teachers may succeed in reaching a certain number of students through techniques that may be very different from ours. How does this happen? And how can teachers more quickly adopt effective practices?

There was both an issue of practice comprehension (and reflection on training) and a need to go beyond the results obtained on individual variability to identify commonalities and find elements that could be modified (and how to do so), without losing sight of student learning.

\section{Enlarging the research scope}

The research discussed above led us to analyze teacher practices in terms of student learning, taking into account that these practices consisted of the exercise of the same profession of "teaching."

We thus broadened our research in two ways. First, we abandoned the exclusive link between in-class practices and intended learning to enter the universe of the profession. We chose the following option: To analyze and interpret practices (and to perhaps train them), we could not ignore the face that these practices, while having student learning as a goal, concerned the sole exercise of the teaching profession, which could not fail to induce specific choices. This represents a significant change of viewpoint for the researcher. The second enlargement was the 
idea of borrowing from the theory of activity, ergonomic psychology, and professional didactics. This work is far from being finished.

We note that the beginning of this work was facilitated by the fact that we were already using elements of the theory of activity in our didactic approach to learning mathematics (inspired by work by Piaget, Vygotsky, and above all Vergnaud).

Together with J. Rogalski, we designed a theoretical procedure called the "double approach" 25 to study mathematics teachers" practices. The name "double approach" emphasizes the fact that we combine didactic analyses of students' mathematical activities with ergonomic analyses inspired by the analysis of the exercise of a profession. The didactic analyses drive part of the analyses of teachers' actions, and the ergonomic analyses complete them. Additionally, some of us have added elements directly inspired by systems of development of work activities.

From the didactic point of view, the analyses begin with the choice of global and local tasks to give to students. The a priori analyses of tasks thus serves to decode teachers' in-class activities, incorporating the proximity of the predicted tasks and student activities, taking into account the resulting unfolding of activities. From the ergonomic point of view, we consider the activity of the teacher as a subject, and not as an element of the knowledge-student-teacher didactic triangle. The choices for the class and in the class, relative to the diverse constraints tied to the institution and individuality thus come into play. From this perspective, we acknowledge that the activity within a particular class depends on the necessities of the profession within a didactic institution. The choices should not be completely random, and studying them from the point of view of the profession allows us to better understand the reasons behind them.

The didactic/ergonomic double approach to the analysis of mathematics teachers' practices

To analyze practices, and specifically teachers' work for the class and in class, we propose here to take into account both goals (such as student learning, but also student investment ${ }^{26}$ and the non-ignorable, non-temporary constraints that are imposed by the profession of mathematics teacher. These constraints decline as we take into account external factors (institutional, social, and personal) as well as different scales of work.

We develop the double approach by acknowledging the complexity and coherence of practices (De Montmollin, 1984). This framework is represented by multicomponent analyses and levels of organization that are recomposed, keeping in mind that is it these re-compositions that reveal what we seek. These qualitative analyses, based on in-class observations and completed through documents collected outside of class, aim to help us understand the student activities organized by the teacher. They also aim to identify what in a practice is fixed, variable (presenting alternatives), temporary, essential, shared, individual, or able to be enriched. 
A starting point: Analyses in a mathematics class

We analyze the practices of a given teacher based on the teacher's classes and organized activities. Our observed variables are student activities as organized by the teacher, interpreted in terms of the teacher's various choices. This interpretation is deepened through various out-of-class studies that relate to the analyzed class periods and allow us to complete our analyses of the observed variables.

From one class period, analyzed in relation to possible student activities, we identify the first two components of observed practices, which we call the cognitive and mediatory components.

The cognitive component corresponds to a teacher's choices regarding content and tasks, including their organization, their quantity, their order, their inclusion within a curriculum beyond the class period, and plans for managing the class period. It can be deduced based on the cognitive itinerary chosen by the teacher for a few class periods. It also allows us to predict, for other class periods, these types of choices.

Choices corresponding to class events, and to the effective implementation in class of the chosen cognitive itinerary, make up the mediatory component. These choices may include improvisations, speech, student investment and participation, instructions, assistance to students in completing the tasks, identification of their work and the work of the teacher, validations, explanations of knowledge, etc. It also includes paths developed for different students.

These components, inferred based on one or more class periods, are then reincorporated into intervention logic, which goes beyond a single class period, allowing some long-term integration, particularly in terms of tying student activities and learning. This logic also affects the personal choices of teachers, which may be otherwise examined (see below).

Our work on the stability of experienced teachers' practices legitimizes, ${ }^{27}$ to a certain extent, this extrapolation (see Part 2, Chapter 2 ????).

The profession: Integration of professional factors that impact practices

To better define the "profession," three supplementary components of practices were introduced: The personal component, the institutional component, and the social component. They represent taking into account data that are not directly observable in class, but that must be considered for understanding certain choices. They correspond to professional factors.

First, a personal component allows us to give appropriate weight to what we see in class and to integrate it within the long term. The teacher can, in effect, make choices, including those tied to the long term. As, in general, we only observe excerpts from a practice over a school year, we can only have an idea of these choices if we ask the teacher, and even this approach is insufficient. This component serves also to translate teachers' representations, which are tied to their knowledge and experience, as well as the risks they take in the exercise of their profession, and the safety they need. A profession is exercised over the long term, and we cannot consent to efforts that are too great for too long. We access these elements in general through interviews, which are best completed by watching 
videos of the teacher in the teacher's presence (see an example in Part 2, chapter 2). There are clearly aspects in this personal component that are even more specific, tied to a teacher's psyche, which we do not explicitly take into account, although we recognize their importance. We remain in the rational, working with elements that are consciously accessible, which legitimizes some simplifications.

Teachers do not choose the transfers that may emanate from their persons. They do not choose the composition of their classes, or the circumstances. They do not choose their automated actions in advance. They do consciously choose, however, the particular content that they will present and the way they will organize and present it. The conscious rationality attached to these choices leads us to favor the corresponding analyses to the extent that we are keeping in mind teacher education, and a rational form of teacher education. This recalls the choice of the possible student activities as an intermediary to access learning.

But to exercise a profession is also to respect a certain number of constraints that may prove to be more or less contradictory with what we would have wished to do on our own. From our point of view, a teacher is not free. We have defined the institutional component by the nature of the mathematics to be taught, the curricula, the schedules, resources such as manuals, the administration, inspections, etc.

We add a social component that corresponds to the fact that the teacher is not alone in a classroom. Students affect what happens in the classroom as a group and as members of social groups. The teacher is also not alone in the institution, but is subject to pressure, expectations (from colleagues, parents, etc.) and occasionally constraints, which must not be overlooked in our interpretations of in-class event. For an extreme case that we have already encountered, consider the young teacher who is strongly discouraged from having students work in small groups because it "makes too much noise," despite the teacher's strong interest in doing so.

This, then, is our first method of analyzing practices within the double approach framework. This division into components, which are deeply intertwined within the complex system representing practices, and the reasoned re-compositions that it allows has enabled us to advance in our research, particularly in finding action logic, commonalities, and variability. In particular, a teacher's choices of mathematical content are directly implied by the very nature of the mathematics in play, as well as by imperatives of class management, by considerations tied to curricula, to the long term, and to the teacher's own representations and knowledge.

Levels of organization in teachers' work

We have identified a second type of practice analysis, still within the perspective of the double approach. This second type of analysis is more suited for examining variability and individual changes in work. ${ }^{28}$

One aspect of the complexity of teachers' work lies in the connections between different distinct phases of this work. Preparation, for example, is partly independent of the rest of the work, but partly influenced by the anticipation of what will happen in class. It is less constrained, particularly in terms of times, than 
is the unfolding of these plans in class or improvisation, which are regulated by the passage of time. Keeping the class on track does not completely ensure the goal of student success, even if the two are linked. Nor does student success ensure learning, even if they too are linked. Finally, it is not possible to exhaustively describe what teachers must do at each step of their work. ${ }^{29}$ It is similarly impossible to completely evaluate teachers' work, as learning is difficult to measure in the medium term, ${ }^{30}$ and difficult to directly connect to teaching, as we have already mentioned.

The three levels of organization used for this component of the analysis consider the different scales connected to the timeframe and texture of the activities to be analyzed. ${ }^{31}$ They are directly tied to the subjects. These levels (or practice organizers) are:

- A micro level, which consists of studying actions that are automatic. This includes non-prepared speech, basic gestures, etc. (Butlen, 2007). We have also examined the method of writing on the board, which is, in part, completely automatic. Other research has analyzed shifts. We note that within these analyses, we may potentially have access to phenomena that manifest without teachers' knowledge and that may remain unconscious. The teacher may become aware of these phenomena but still have difficulty acting on them.

- A local level, concerning the daily class. This level contains preparations and improvisations, and is the level of all teacher adaptations.

- A macro level, for projects and preparations, ${ }^{32}$ based on individual knowledge, representations, and experiences.

\section{Some examples of practice analyses}

An initial type of results involves the confirmation of the individual coherence ${ }^{33}$ of practices (De Montmollin, 1984) and the fact that they become stable over time. The mediatory component, examined to a certain level of detail,${ }^{34}$ is the most stable (see part 2, chapter 2). Other work on teachers' use of the board confirmed the consistency of each teacher's choices, and the coherence with the chosen method of classroom management (Robert \& Vandebrouck).

Another type of results helps explain the consistency of the intervention of institutional constraints in practices (close institutional components), given the diversity within the other components (see Part 2, Chapters 1 and 4 !!!!).

As we will not return to this topic in subsequent chapters, we will summarize here some previous results concerning practices of experienced teachers that we found to be common in 9th and 10th grades, as well as results from beginning teachers that demonstrate the use of levels of organization.

Examples of consistency in 9th and 10th grade class periods devoted to exercises We will only give a summary of the results of these studies ${ }^{35}$ (Robert, 2005b and c). 
Teachers favor in-class work that exclusively focuses on the new mathematical concepts being taught. This type of work does not involve much exploration of the field of problems solvable with the associated tools. Effectively, the necessity of progressing through the topic leads teacher to propose tasks that are relatively close to the lesson, that require standard applications of the concept, which must have been already seen. This leads again to favoring "decontextualized" meanings versus "contextualized" meanings. At the same time, there is little explicit maintenance of prior knowledge. There are rarely occasions of reorganization between old and new concepts. Furthermore, students are only rarely and briefly confronted with uncertainty on what they should do, which leads to minimizing student questioning of what should be used and autonomous linking of concepts.

This takes place through organized in-class events and by teacher interventions before and during student activities. We identify an unequivocal orientation of students' activities toward the desired new knowledge. This orientation is particularly enabled by a precise and rapid (indeed, immediate) consideration of these activities, with constant guidance and little time for autonomous work that is not on the final calculations. These calculations are completely outlined for the students, and form the major part of students' in-class work.

The resulting activities thus relate to tasks that, if not originally simple isolated tasks, become isolated. These tasks relate to the relevant chapter, without many adaptations of the concepts to be used. There is rarely need for structuring knowledge as an action for students, as the teachers handle this themselves. In these conditions, there is also no need for delegating control to students.

We identify therefore a certain sequencing of student activities on a concept in relatively independent moments. Students apply the tools, one after another, independently. They only need the (stacked up) tool concepts corresponding to the lesson and inspired by the teacher's subdivision of activities. The development of the dynamic between lessons and exercises can be limited on scope. It is thus the organization of student knowledge that will be one of the first victims of this time constraint.

The constraints shared by teachers of the same grade in similar establishments (schedule restrictions, curricula, effect of instructors and inspectors, substantial heterogeneity, class composition, etc.) act as if to lead to shared practices, in terms of the mediatory and cognitive components, even if there is leeway accorded to teachers that is not applied in the same way.

We cannot be sure that students' knowledge will be partitioned, ${ }^{36}$ as students can learn things that are not explicitly taught to them (and that are therefore intended for them, more or less implicitly). But we can still ask if the common complaint of observers of the lack of "certainties" among students may come from this type of classwork. This is reinforced by a common complaint from students: "Just when we start to understand we switch chapters." One question emerges: Are there alternatives to this type of choice? 
Examples of analyses of practices of beginning teachers

The study of transitory practices of beginning teachers can also illustrate this type of practice analysis. Beginning teachers ${ }^{37}$ (called PLC2 in France) develop practices that evolve during their first year. We call these practices "transitory," as they are not yet stable. They are usually complex, however, and we assume that their coherence is developing, due to their previous experiences and knowledge.

These beginning teachers are led to adopt a new position, that incorporates their personal component and that is tied to the exercise of a new profession, in an actual establishment. This leads them to become aware of constraints and leeway of their new profession: "Not everything is possible, either for everyone or for each person."

Every day, in beginning teachers' classes, we see evidence of difficulties in recognition of students and time management (the mediatory component). It is possible that the mathematical project of the class period may be central to the detriment of students, or that consideration of students may be focused on to the detriment of the mathematical project. It is as if some beginning teachers are obsessed with the reactions of the class and the concern that all students follow along, while others forget that it is to their students that they are teaching mathematics, and even display ignorance of mathematics for students.

At the level of the cognitive component, the project developed by beginning teachers is often fairly local. It covers a maximum of several class periods, and does not always fit into a coherent whole for the year, particularly in terms of the mathematical plan (Margolinas \& Riviere, 2005; Bloch, 2005).

Other complementary elements were proposed in a study by Bloch (2005), who suggested that the PLC2 teachers did not only lack the means to organize their lesson content, particularly introductions, but also had specific difficulties regarding students' mathematical work that could take many forms. It is, paradoxically, a lack of awareness of the necessity of having transitory constructions serve as intermediaries for students, and sometimes even a lack of awareness of the necessity of the construction of meaning. Bloch uses this to support the idea (previously used by Lenfant, 2002, in algebra) that certain beginning teachers have so internalized some mathematical concepts that they no longer see the difficulties. They are not aware of the fact that giving a rule, even with commentary, is not sufficient for all students to learn it. They may also expect formal proofs too quickly while students can proceed though more pragmatic processes. Finally, Bloch proposes giving PLC2 teachers ways to "have students really do mathematics," particularly by identifying the concepts in play, elaborating situations in which the topic will arise, and learning to handle them. We will return to this.

Finally, these unstable practices of beginning teachers lack sufficient operational mental images to enable nuance and adapted improvisations. Beginning teachers have incomplete or skewed images (Chesné, 2006). Even if their images are not what we call "deformed," they may lake depth or a hierarchy in a desire to "do well" by following their training. They may temporarily erase, as much as possible, the personal component and spontaneous reactions (Chesné 2006). These teachers 
thus do not involve themselves completely as such, with consequences on students who do not have a truly engaged teacher in front of their class. Other teachers may have "caricature deformations" which overly focus on the individual relationship with students or their activity, which may be more or less mathematical. Others overstress following the mathematical project they have decided to adopt, the presentation of knowledge and the course of exercises.

We hypothesize that, unable to rely on automated processes, routines, or overall depth concerning either mathematics or students prevents these beginning teachers from leaving the local level, which then becomes all there is. With a lack of connections to the micro and global levels, there is an overload on the local level.

The case of newly certified teachers, particularly those in their first positions in the Zone of Educational Priority, has led to recent studies (Coulange, 2006) that show the diversity of the potential effects of teacher training on those trained, relative to their personal component, their first position, and the details of their training. One question arises: Are there pre-existing factors that could lead a sizeable number of beginning teachers adopting certain practices over others? We will return to this in the last chapter.

\section{RETURN TO STUDENT ACTIVITIES - A METHODOLOGICAL POINT OF VIEW - SOME QUESTIONS}

Even if each study chooses elements of this general methodology to adapt, the work to be conducted in order to study the teaching of a given mathematical topic can be divided into six "acts," which include better understanding learning and teaching practices, and describing alternate strategies to try. These acts are clearly non-independent, but may be completed in various orders.

\section{Act 1: Determining the relief map corresponding to the concept}

This phase of research leads to examining epistemological or historical studies, as well as didactic studies. The goal is to define the mathematical details of the concept, to characterize its role in the curriculum (and its potential evolution), and to synthesize students' identified difficulties. This can be enriched by analyses of manuals or other resources.

\section{Act 2: The studied or intended teaching scenario}

It is clearly the reference to the relief map that allows us to appreciate the intended introduction as much as the dynamics between the lesson and the exercises and/or the richness of tasks. This is a difficult task, as it often relies on many inputs. Comparing different scenarios may help. 
ALINE ROBERT, CHRISTOPHE HACHE

Act 3: A priori analysis of specific tasks, from problem statements presented to students

The subsequent chapter on the analysis of manuals illustrates in detail an example of these analyses.

Act 4: Analyses of in-class events, based on observations or on video or audio recordings

Analyzed class periods are often filmed and then transcribed. The camera is placed at the back of the room, centered on the board, with the teacher as the principal actor. The students are rarely seen and cannot be heard well, but the teacher often repeats their statements.

To complete the corresponding types of analyses, we have established a rubric for studying classroom events (described in detail later on). One of the variables is the level of detail of these analyses. Depending on whether we examine speech phrase by phrase or more finely, the information we collect is different. For example, to understand the dynamics of interaction phases, it may be interesting to examine language markers (see following chapters).

We compare a priori analyses of problem statements, the work conditions in the classroom, and all verbal exchanges to reconstitute students' activities. We go into more or less detail depending on the specific research question.

We first take into account the established chronology. Beginning with the $a$ priori analysis of the problem statement, we list student tasks and their length as they are encountered throughout the lesson, with reference to the a priori listing.

We thus note the task format (individual, collective, etc.), the output of the task (simple research, written work, group written work, etc.) and the types of tasks (researching, writing or speaking, listening, composing, recopying, etc.). Task types and task formats determine the nature of the work, independently of the work content. In particular, moments of silence by the teacher are taken into account as indications of an attempt to delegate the task to students. In this way, students may, to a certain extent, research, discuss, write, listen, recopy, get help, get corrected, or even be encouraged, on a single task. The nature of their work (for example, investigating as a group, writing out a response individually) gives weight to and indeed modifies the application of the knowledge induced by the tasks.

We then compare the tasks and subtasks worked on by students and the teacher's contributions: questions, rephrasings or answers, help or explanations, identifying and applying student work, presenting knowledge, generalizations... silences. Particular attention is paid to teacher assistance. Recall that procedural assistance can reduce the task to be accomplished, but can also allow students to apply themselves to work. Constructive assistance can allow students to construct new knowledge based on their own work. This corresponds to use by the teacher of what we model under the generic term "PDZ." From the students' point of view, procedural assistance may already be constructive, and constructive assistance may remain procedural (cf. connection to knowledge of students in a ZEP). Generally, 
all commentary added by the teacher, whether on the mathematics in play or on student work, is an important element of our analyses of in-class events (cf. meta). They can reveal what improvisations, choices, and thresholds the teacher selects, relative to what was expected. This in turn can reveal both possible student activities and teachers' logic. In terms of the phases of knowledge presentation (or "lessons"), analysis is a little more difficult, especially if students are silent. Moreover, it is imperative to be aware both of what precedes the lesson, and what immediately follows it. The lesson is often illustrated by simple examples or immediate applications, to establish the potential real or artificial link from activities before the class period to the class period.

We replace the analysis of tasks by the analysis of units, which may be present or implicit in the lesson. Possible units include definitions, theorems, properties, propositions, demonstrations, examples, commentary, illustrations, diagrams or drawings, and applications.

We then investigate these units in the same way as before. We examine particularly their existence, the length of time spent on these knowledge presentation phases, the order and placement of the presented content, and the manner in which the different comments are introduced, as well as the moment they take place. This shows us the potential way the professor introduces the object and tool aspects of a single concept (the corresponding theorems or methods).

We seek, if possible, the apparent role of improvisation and conversational exchanges during these phases, as well as students' apparent activities. Writing on the board, and differences between what is written and what is said, may also be analyzed (depending on the study). Similarly, the degree of mathematical formalization may or may not be analyzed.

It is interesting to analyze how the teacher asks students to use the lesson (and to work on it), and to note the quality of the references the teacher makes to the lesson. It is as if for some teachers the lesson serves both as a reminder for the class and as a catalog of knowledge to use.

It may be interesting to compare teaching manuals with teachers' lessons, as well as the use that is recommended for students.

\section{Act 5: Reconstitution of students' activities, links to learning, initial questions}

The preceding analyses allow us to reconstitute the traces of possible permitted or encouraged student activities. Possible activities are those that we can estimate were done, at least in part, by many students during class time. Often, we are otherwise led to distinguish a maxima activities and a minima activities (cf. I). By contrast, since students work autonomously on a computer, accessing their activities is less problematic (even if in actuality no one can access the effective activities themselves). We thus speak of the observed activity (even if only the actions were observed) and we have developed several methodological ways of accessing these activities (by direct observation or thanks to trace files; see part 3, chapter 3). 
That is to say, if it is difficult to analyze teaching in relation to learning, it is even more difficult to have legitimate evidence of it. We are well aware that a blank sheet is not always synonymous with a lack of learning, that an apparently trivial variation of a problem statement can affect student performance, and that there may be a large gap between what a student writes and what the student has understood and retained, or is in the process of learning. Tests, for example, provide very limited evidence of learning, polluted by social and affective factors, and tied to the teaching contract and to the necessity of having a certain level of success in a class.

Our analyses thus do not permit us to do other than pull out relatively contextualized relationships between problem statements and in-class events on one side, and student success on tests on the other. We will, however, have to content ourselves with this in certain studies (see part 2, chapter 4).

Note that, more generally, our theoretical framework does not allow us to form precise hypotheses concerning these teaching/learning relationships. It is perhaps on the edges, with students having difficulties, for example, that the compensations that may influence some students are no longer effective, or that there may be some threshold effects. For example, researchers (Castella, 1995, 200) have shown that some students can construct knowledge without their having been explicitly taught. Are there sources of differentiation here? Are they individual or social?

At most we can give several hypotheses (see Appendix 2) on extreme cases, inferred from the general theories that we use for inspiration. First, a completely lecture-based lesson has a strong chance of preventing many students from constructing knowledge, due to insufficient student activities. A lesson with no period of knowledge exposition may also prevent students from learning, due to a lack of occasions for transforming activities into knowledge. Similar problems may be found in a lesson that contains only simple and isolated tasks, due to a lack of non-immediate activities.

\section{Act 6, the logic of teachers' actions and analyses of their practices}

This last act consists of reconstituting and recomposing, for a single teacher and then potentially several, the components that we have distinguished, keeping track of the organization into levels.

The action logic combines the cognitive and mediatory components, to better identify what can lift the constraints we take into account.

Using multiple analyses of a single teacher or of several teachers, we look for common factors in overall content choice. For beginning teachers, for example, the extra burden of the local level has led to calling on levels of organization to interpret the records. Determining professional groups (such as gender) leads to working both on components and on the levels of organization.

Diversity between practices is often expressed through logic of action (on the local choices of tasks and class progressions).

These are, for now, inferences based on initial results that lead us to suggest variabilities (what can shift, and at what cost). 
A number of chapters of this book illustrate this last act.

\section{Conclusion: further questions - toward the longterm?}

We indicated above the open questions concerning appropriate indicators for studying the speech of teachers in class. These indicators can be more or less finely grained and can consist of linguistic markers or the format of exchanges. But these analyses of in-class events, based on analyses of tasks (exercises, lessons), remain essentially local. They cannot completely reveal events over the long term, although learning itself takes place over the long term. There is also no "rung" to better appreciate student activities and teacher motivations.

Teachers often do not say or do all that they intended. This feeds a double discussion of the necessity of their choices and omissions, and of the alternatives. The elements analyzed during several lessons leads to larger questions concerning other potential exercises, the lessons, the curriculum, the class, the specific teacher, the establishment, etc. Focusing on these questions allows us to go back up to the global level.

The study of lesson plans can certainly reveal part of the mathematics commonly used by students, in terms of dynamics within the project, between the lesson and the exercises, and between the meaning and the technique. They are revealed through the order in which the different parts are presented, the expected lengths of the different phases, and the quantity and variety of the tasks. But the difference between what is predicted and what actually happens, as highlighted by this chapter, is too great for this to be sufficient.

When possible, examining student work for a given chapter can certainly allow us to compare in-class events with evidence of learning. However, this is very time-consuming, as all events must be examined relative to the test. Some studies have begun to devote such resources, although they are limited in scope or in the number of parameters.

We retain, from this theory of learning and practices, the intermediaries chosen to have access to it, with the importance of coupled links (statement and in-class events) as precious indicators of factors that can vary in student and teacher activities. These activities can be understood through a priori analyses of expected applications of knowledge from a problem statement, compared to the applications provoked or allowed during the class. These latter applications are approximated by the work that the teacher puts into place for students. These analyses of possible activities are sometimes completed by studies of observed effective activities. More general studies of the mathematical concept in play, from the scenario in which the classes take place, and from the institutional, social, and (for the teacher) personal context, reveal recompositions respecting the complexity in play. 


\section{POSITION RELATIVE TO OTHER STUDIES}

Our investigation relates to the effective class and individual subjects. We indicate in this section, in a necessarily schematic and summary way, several general characteristics of this theory relative to other foreign or French didactic theories. Note that it is impossible in a few pages to be either exhaustive or complete, and that we have selected several examples for clarifying our proposals.

We will see a large initial difference concerning whether to adopt a "stricto senso didactic" point of view, where subjects play a generic (or indeed epistemic) role, and are therefore all considered equivalent. This is not our viewpoint.

\section{Concerning students and student learning}

A certain amount of anglophone research is directly inspired by the theory of activity for analyzing class periods (Christiansen \& Walther, 1986; Hiebert \& Wearne, 1997; Stein, Grover, \& Henningsen, 1996; and others). We also find that for them, the task is the starting point for the activity, and remains external to the student, while the activity is what the student actually does, and what influences learning. They give particular importance to in-class events for analyzing student activities, and are not content to study the tasks students are proposed. However, the methodologies used are different from ours, and the activity is analyzed more in terms of overall applications of mathematical steps than applications of precise skills such as conjecturing on an open-ended task, calculating, or reasoning on the nature of a problem, internal or external to the mathematics. Here is a list of four such types of tasks given by Sullivan, Clarke, Clarke, and O'Shea.

- Type 1. Involves a model, example, or explanation that elaborates or exemplifies the mathematics.

- Type 2. Situates mathematics within a contextualized practical problem to engage the students, but the motive is explicitly mathematics.

- Type 3. Involves open-ended tasks that allow students to investigate specific mathematical content.

- Type 4. Involves interdisciplinary investigations in which it is possible to assess. Great importance is given to open tasks and to associated investigatory steps. This corresponds notably to research developed in relation to the NTCM (see below). However, characteristics tied to affective or psychological dimensions are often introduced, which partially direct the analysis of tasks and activities differently from our analyses led by knowledge stricto senso. Some examples are "attention" (Mason, 2003) or "challenge" (Jawarski, 1994).

We return briefly to francophone research. In the Theory of Didactic ${ }^{38}$ Situations (TSD), when in-class events are analyzed, references and the comparison in the light of which we report observations are the model of mathematical learning defined from fundamental situations and the environment. Fundamental situations, at the heart of the theory, model a didactic procedure that in some sense forces students to use the mathematics to be acquired. The corresponding problems are developed from the deep meaning of concepts, 
identified from the question: What are the concepts for? Students have no other option but to use them, on the condition that they play the proposed game. Moreover, it is within the problem that they find the elements that allow them to determine if their work is correct. They do not need to wait for the teacher's input (see the "puzzle" example).

From the perspective of in-class events, Brousseau (1998) particularly highlighted the interest of the sequence of phases of action, formulation, and validation. He introduced the concept of the didactic contract that represents the potentially implicit expectations of the teacher towards the students and vice versa. We can thus gauge a priori the different situations (including ordinary situations) proposed to students that use the tools initially conceived for describing "ideal" didactic situations. The a posteriori analyses allow us to compare the gaps between what actually happens in class and the a priori analyses. This involves verifying if the tools the students must use to solve the problem are available to them and are well within the environment. If the situation is predicted to be a-didactic, in order to introduce a concept from a "good" problem, for example, we verify a priori that there is no need for the teacher, that the intended knowledge is necessarily and uniquely at work, and that the situation is within students' reach. We verify $a$ posterori that the actual lesson respected the expectations. Based on the characteristics of the in-class events and the predicted tasks, the analyses explicitly confirm or reject the gaps between what took place and what could have been predicted based on the model of learning within the TSD. We can invoke the point of view of a certain possible theory of learning for analyzed situations: that analyses carry inferences on learning through the intermediary of the model (Brousseau, 1997, 1998).

For our part, we seek to measure the gap between the activities of students applying their knowledge (during its acquisition) analyzed a priori, and the activities that may actually have taken place during a regular lesson.

First, the description of these activities is made in reference to large dimensions that do not correspond to a constructed theoretical model, but only to large categories of variables influencing learning and depending on teachers. We should note at this point the importance of the chronology and the corresponding details of the assistance provided by the teacher in our analyses. The chronology and finegrained analyses of speech seem to us to be often absent from analyses of the environment, where the situation is analyzed as a whole from the start.

Second, we can work equally well with short sequences as with longer ones. This is indispensable at certain moments during the research. In effect, our basic unit (the problem statement/in-class event couple) is smaller than the "situation" in the TSD sense that it replaces. We can however note that it is "of the same order" in a certain sense, as it combines content and management. But the concepts of tasks and activities do not explicitly appear in TSD. They are replaced by situations proposed to students divided into possibly a-didactic phases, referring to students' single mathematical work within each phase. The dynamic of reference situations refers uniquely to the application of the expected mathematics, made indispensable 
by the proposed problem and which is with internal methods of control for students. The individual variabilities therefore have no place.

Finally, we give the same importance in our approach to the introductions of concepts as to the rest of the instruction of a concept. We do not find a focus on fundamental situations or the tool/object dialectic. We also do not find the implicit increase of the introduction of concepts for the construction of knowledge by students.

The anthropological theory of didactics $\left(\mathrm{TAD}^{39}\right)$ is another different theory. It pertains to the didactic of elements inspired by an anthropological vision of man in the world. From mathematical decision theory, we obtain systematic and systemic ways to establish an exhaustive definition of an institution's mathematical provisions in terms of one or more concepts: curriculum, manuals, or even more or less complicated mathematics. The "provisions" are everything available to students and teachers in terms of types of tasks, (legitimate) justifications, theories, etc., without taking into account conditions of instruction unrelated to the mathematics. In particular, it is not the problem statements of the exercises that are analyzed, but the types of tasks that they illustrate and that are extracted from them, independent of the specific activity that the students have to do on the task in question, which we have not given ourselves the means to determine. This leads to identifying types of elementary tasks as kinds of units that can appear in exercises, manuals, or lessons, and that serve to distinguish the instruction from the content, understood in terms of gaps or evolution. Each type of task is associated with one solving method or technique and to various justification possibilities (which may or may not be present within the analyzed elements). The whole, which can range from one concept to several chapters, and which can cover one or more curricula, is organized relative to the elements of the corresponding mathematical theories and the evidence of the transposition of this knowledge into teaching content. It is thus not in this sense that the word "task" is used in the presented work (Chevallard, 1992).

\section{Concerning teaching practices}

Many anglophone studies involve teacher beliefs, but do not introduce the occupational dimension. For example, the model developed by Schoenfeld (1998) characterizes decisions and actions as a function of one's knowledge, goals, and beliefs. These factors are clearly marked by the content that is considered. This model is used to characterize a moment within the teaching activity, included within the level of student interaction and aiming to also predict the behavior of a teacher whose factors have been previously determined, which underlies the existence of certain invariances. In addition, a certain number of studies now examine collective systems that involve communities of practice that serve more to study potential evolutions in these practices than to analyze them in relation to student learning (Wenger, 1998).

In France, in research inspired by TSD, Margolinas (1995) and others presented an analysis of teacher knowledge and its role in class, organized in levels 
structuring the environment. For Margolinas, working (for a teacher) is "putting in play" knowledge of different levels of mathematics and student. Studying the work consists of examining this knowledge and its interactions between these levels and then imagining ways to have this knowledge acquired.

Much of this knowledge concerns the way in which the professor understands the content within a curriculum, develops exercises to give to students, and interprets student knowledge. This type of fairly large investigation does not seem to us to take into account the chronology of the lessons, or the way they unfold in class, which become secondary. The manner in which actions, such as taking up a student's idea or offering help, are taken, are thus not described and have no place.

From the TAD viewpoint, the actual lessons that take place are systematically related to the didactic organizations that are developed independently of classes, teachers, and concepts (Chevallard, 1999). Thus, different points in the lesson (introduction, work on the technique, etc.) are taken into account. However, in these studies, the import of the effected lessons and the specifics of individual subjects, which we take into account through the intermediary of the analysis of effective activities, seem to us to be discounted.

Finally, more recently, researchers have developed a model of the action of the professor in class, which has been expanded into a model of the joint professorstudent action within the framework of the compared didactic (Sensevy et al., 2000). The decomposition of the teacher's action into four dimensions, and the systematic consideration of the mesogenesis, chronogenesis, and topogenesis, also do not seem to us to be well adapted to our project of realtime introduction and individual and conceptual variabilities. The explicit theoretical references to cognitive characteristics of student knowledge and to effective student activities are not called upon.

In this step, working consists of bringing activities into play, and analyzing consists of studying the activity (what is thought, said, unsaid, done, undone, etc.). We are less interested in knowledge than in its application, and we do not reduce teachers' activities to professional gestures, even if it is interesting to introduce levels tied to the temporality and grain of these activities.

These different viewpoints clearly have consequences on teacher training and corresponding research. We will return to this in the conclusion.

\section{Concerning professional didactics}

Although our work falls within the general double approach framework, it borrows more or less specific elements from professional didactics (Pastré, 2005a \& b). We have allowed ourselves to be more directly influenced by research on the integration of technology into teaching practices.

For example, in some research, we study the teacher's activity in a specific situation of integrating technological tools in the classroom. Our approach is directly inspired by professional didactics. In these studies, the dialectic between "productive activity" and "constructive activity" is introduced to clarify that the teacher acts to transform what takes place by contributing to students' activities, 
and that the teacher is also transformed though a longterm development process (Pastré \& Rabardel, 2005).

We would like to add in this section several difficulties that arise from these borrowings. They are first tied to our difficulty in defining schemes and integrating the concept of competence. They are also tied to questions of expertise. There is not always agreement in mathematics on the orientation of the the teacher's action. There is far from being a universal definition of "good ways" to teach, nor common adoption of reference models for analyzing practices. Moreover, the test of a teacher's practices is not made on learning, the ultimate goal of practices (or only partially, by the intermediary of proofs for which everyone agrees to underline the insufficiencies inherent to the complexity of such an evaluation). By contrast, the teacher, and even the educational inspector or other colleagues, can verify that "the class functions" or that the students succeed on tests. It is not the goal of the action that is easily and directly tested, but only a partial subgoal of student investment, which is a no doubt necessary condition but which can not reveal learning. We wonder if a multi-expertise is not necessary in this professional domain.

The individual/invariant/generic relationship, and the differences between the action sequence and the significance for each and invariants, are unique to the teaching profession, as is working in a human open dynamic environment. Complexity, variability, and unpredictability make the subdivisions that allow us to locate the execution of the action, and no doubt require several simultaneous and interrelated approaches, problematic.

Finally, the analyses of actual work structure many ergonomic studies. In the studies on teacher practices, this type of analysis is difficult to perform, given its complexity (the presence of students, the difficulty of the evaluation). Consequently, many teacher trainings, for example, are inspired by the work desired by the trainers, without always being anchored in the real work of teachers, contrary to many formations inspired by professional didactics.

\section{$I V$-Technology in education: details on the case of work on the computer}

In the case of computer-based work, a preliminary examination on the use cases of technological tools is specifically relevant, to the extent that the discussion of the longterm of computer-based lessons with classical lessons is a factor in student activities. The a priori task analysis should be completed by an equivalent analyses of environments, assistance, and possible feedback. Along with teacher assistance, they can modify the expected possible activities.

For in-class events, the length and nature of student work differ from one type of tool to another. Work is, however, often individual, and students have a priori a lot of autonomy. They can have different work progressions with different amounts of time spent on the proposed tasks. They can also benefit from individualized feedback, and can discuss with partners or with the teacher. There is thus in general more material to observe than during traditional lessons in which the rhythm is often dictated by the collective progression of the class. Students can thus be 
followed individually. We have access not only to their possible activities, but to their actual actions. The reconstitution of the activity is this closer to reality than it is with classical methodology. We can better determine if constructive assistance serves its purpose, and thus to go towards effects in terms of learning. However, there is a loss of generality, as only a few students can be observed.

Learning specific to the use of technological tools, combining mathematics and knowledge of the tools themselves, appear in particular in students' use of openended software (dynamic geometry, spreadsheets, etc.). They can justify using a theoretical approach centered on learning. It involves using an instrumental approach (Rabardel, 1995) specific to the mathematics (Artigue, 2002). This will be discussed in part 2, chapter 4 in terms of the introduction of spreadsheets in classes.

Finally, some assistance from the teacher is uniquely tied to the computer environment and the manipulation of tools. It is not found in the traditional paperand-pencil environment (except when non-technological tools, such as a compass, straightedge, etc., are used). We introduce in some work a third type of assistance (after procedural and constructive assistance) which we call "manipulatory" (part 4, chapter 1).

\section{$V-$ Conclusion}

We could diagram the main dimensions that allow us to distinguish researchers' choices.

An initial source of diversity is the connections maintained by didactic studies to the situation (the time and the problems found in the countries involved), to the terrain (the school, the students, and the teachers), and teacher trainings, in relation to the institutional conditions that are imposed on research. In concerns the position that is adopted concerning the links between the research and the instruction prescribed in a given area, the curricula, the instructions, but also the cultural or social habits. In a word, does the research have a prescriptive or prospective impact? Do they provide diagnostics or propositions to be tested (or not)? What variables are introduced? In relation to the problems to be treated, in what theoretical or conceptual framework does the research take place? What is its role?

Some studies fit directly in a given educational system, with goals of acting on the system. We note particularly anglophone studies attempting to increase the effectiveness the NCTM standards established in the 2000s to improve mathematics instruction in the US (and in other countries that have adopted the same types of standards). Analyses of tasks that are then produced, for example, seek to translate not the epistemological characteristics of the mathematics content but student skills to be accessed (looking for a solution, conjecturing, writing up a solution, etc.). Other researchers work with the same goals as, and even together with, teachers. They have analogous goals tied to the reality of what takes place in class. They declare explicit objectives of transforming instruction and improving learning (Boero). Still more look for cultural or social sources for the groups 
involved within the mathematics content (Radford, 2010). By contrast, some researchers focus more on characterizing universal forms that are necessary for mathematics instruction, tied to content (fundamental situations) or to understanding how knowledge evolves and diffuses, establishing for example a structure of knowledge (Brousseau, Chevallard, Sensevy in France). Still other researchers attempt to clarify how teaching and learning are tied, and what are the resiliences, the invariants, and the diversities, and beyond this the local or general situation (Vergnaud et al.). These latter researchers call more on theoretical frameworks, which are often models serving as reference to research, to choose before studies to adapt or develop. The first types of researchers work with local or general theoretical frameworks (or a mixture of the two) that seem appropriate to begin working on the questions to be treated. Other researchers remain working on empirical or positivist studies. In France, research on mathematical didactics was able to develop with some independence of the situation. From this, theoretical frameworks from anthropological theory to be adapted to didactics, from game theory as a model of learning, from Vygotsky and Piaget's theories applied to the theory of activity, were developed, ahead of contextualized research.

Finally, research on training mathematics teachers in teaching or mathematics are more or less associated with didactic research. In some countries, this is even the origin of the didactic question. Here again is much diversity. We will return to this in the last part of the book.

Another distinction, which is not unrelated to the preceding and to theoretical choices, relates to what is taken into account in research in terms of students and teachers, and the corresponding variables that are introduced in studies, in connection with the theoretical or conceptual frameworks adopted and their definitions. A student learning mathematics and a teacher teaching mathematics can be "approximated" and analyzed in several (non-exclusive, non-independent ways):

- As an "epistemic" subject (studied in relation to the invariants that characterize the subject's evolution, and in terms of the subject's function or role—student or teacher).

- As an institutional subject (studied in terms of "subservience" as a function of the institutions to which the subject belongs).

- As a social subject (related to both the subject's sociocultural origins, particularly for the student, and the subject's profession, particularly for the teacher).

- As a psychological subject, with multiple points of view.

- As a cognitive subject (studied in terms of the subject's development, in terms of learning potential for the student, and practice enrichment potential for the teacher).

- As an affective subject (studied in terms of emotions, tastes, self-confidence, etc.).

- As an individual or psychic subject (with a personal history, representations, knowledge, character traits, etc.). 
Even the name of the corresponding scientific field in different countries can be revealing.

One final dimension is tied to the consideration of quantitative aspects and validation in studies and, more recently, to the role given to evaluations. Do we work on individuals, on a few classes, on a large number of classes, on a category of students (possibly characterized by a "generic" representative), on a professional group (such as middle school teachers)? Is there a place to validate the conclusions, and if so, how?

This dimension is effectively tied to the mode of validation predicted in the studies, and to the theoretical frameworks. A strong coherence with a certain framework can serve as auto-validation to certain studies. This, the role given to experimentation, and more generally to all data analyses, varies widely in relation to the theoretical model and with the potential discussion of or the search to enlarge this model. If used to explore new fields of mathematics instruction, experiments serve to collect data for which the model prescribes analyses ${ }^{40}$. If not, if the research is led by questions that do not fall directly under a model, or that contradict it, or that borrow from general theories, experimentation can also serve to establish commonalities, hierarchies, causality, or dialectics contributing to increase or renew the understanding of the phenomena.

For now, research in France is mostly clinical. It is qualitative, and, depending on the case, often validated by comparing predictions to outcomes. There are many people who imagine changing the scale of this research. Here there is another link to the objectives. Research that is very linked to the objectives to be obtained for student learning cannot go without quantitative evaluations, even if we know the main limits: uncertainty on the causes of learning, on the moment to evaluate, on the tasks to propose (not to close or too far from the information learned), etc.

International evaluations are not always associated to didactic research. However, their results allow us to revise quantitative perspectives thanks to powerful statistical tools and extraordinary improvement of ways to improve data collection and associated processing.

Depending on the role given to the situation and the terrain in studies, and according to the types of situations considered, some variables are more or less imposed on researchers. Thus, in France, the analysis of tasks in terms of types of tasks, techniques, technologies, and theories is more adapted to an overall, more or less exhaustive analysis of the knowledge content to be taught. This analysis can critique and even break from curricula, concerning primarily institutional subjects (in various institutions. Our a priori analyses (presented above) are more adapted to understanding possible student and teacher activities within a given curriculum (concerning epistemic and cognitive or even social subjects). They require placing task within an overall cognitive itinerary, with reference to a conceptual field (or to a level of conceptualization). From this viewpoint, curricula can be contested through arguments that are not only epistemological but also tied to subjects (particularly students). Studies that involve teachers as psychological subjects (from a certain point of view) are also different from studies involving institutional subjects only to the extent that other variables are taken into account, such as 
leeway for constraints and their investment, consisting of choices, alternatives, etc., and not only constraints. Still other research very tied to the terrain has developed methodologies specific that involve diverse communities of practice and discourse created with researchers, teachers, and teacher trainers.

That being the case, researchers in mathematical didactics all have the goal of first taking into account the specifics of the mathematics to be taught - to the extent that they share the fundamental postulate of the importance (and specificity) of the nature of the content in play in the learning. But the descriptions of the mathematics necessarily depend on the nature of the students and teachers considered, and even the nature of the relationships between learning and teaching in which we are interested. Several types of relationships between teaching and learning have been studied. This, if all agree to extract, from the history and epistemology of mathematics, curricula, and their evolution, a description of the content to be taught (concepts, chapters, domains, or mathematical fields, then the very modalities of these descriptions depend largely on the didactic project and the studied "subjects," as we have already discussed above for francophone research. Some other researchers place a lot of weight on the language developed in class, and specifically study the communication that is established. Others insist on the semiotic analysis that can be done, notably from the moment instruments become involved.

If the problems are approached quantitatively, we can extract important variables that are susceptible to revealing the recorded variations. They can be used both to describe the modes of investigation and to analyze them. There is no question of looking at individual differences, tied to taking into account individual subjects. There is also no question of attributing to a specific result an interpretation that goes beyond the variables selected. This is no doubt the origin of the week impact of some international evaluations, which, wanting to include to much, do not leave much room for interpretation.

By contrast, if we wish to understand the more individual games played in class, the margins that remains at the interior of a given system, then the choice of variables selected and the types of subjects studied may be different. If, for example, we want to introduce the student as a "cognitive" subject, and the teacher as an individual subject, then we need descriptions that are not limited to the mathematics that is structured and analyzed in relation to the knowledge only. It is necessary to given, in the descriptions of content, ways to describe potentially unexpected student difficulties, and, more generally, the learning that evolves. It is also necessary to give methods to analyze what takes place in class, along with what was predicted. The concepts of "conceptual field" and level of conceptualization are a response to the first expectation, while the analyses of inclass events respond to the second. It is also necessary that these latter analyses can reveal what we are looking for. Thus, the a priori analyses of tasks in terms of adaptations were introduced specifically to allow for studying the outcomes of the predictions in class and to better appreciate subjects' activities in response to tasks processed in class. 


\section{Concerning other disciplines}

In France, various types of didactics have been developed with different histories. Some come from teacher trainers (didactics of the French language), and others from universities (didactics of mathematics and physics). Some interdisciplinary studies related to science (particularly mathematics and physics) begin to be conducted. This usefully increases the spectrum of questions that are posed. But this is another story.

\section{APPENDICES}

\section{Appendix 1}

Frameworks, registers and points of view

We adopt the Douady's categories (1986): a framework corresponds to some mathematical field (or domain), in which a given notion is introduced, not alone of course. It is characterized by some fundamental axioms, implicit or not, a corpus of definitions (objects), theorems, and propositions and a set of problems that can be resolved within this field. For instance the middle of a segment $[A B]$ can be studied in an analytic way (involving the analytic field), geometric way (geometrical field), and so on.

According to Duval (1995), we call "register" a precise way of writing mathematics, using a given formalism, introducing so a semiotic view. For instance to work on decimals numbers, some can choose such writing as 4,567 , or $4+5 / 10$ $+6 / 100+7 / 1000$ : these two registers differ and inside each of them, the treatments (operations) are not exactly the same.

The points of views refer to different ways of tackling a problem, leading often to different strategies. For instance one may consider the intersection of three lines as a point belonging to each of them, or may consider that the intersection point of two of them belongs to the third one, or may look for a transformation such that the three lines are the image of three other intersecting lines... Each point of view induces another strategy to tackle a problem. They differ from field or registers because they can occur inside of the same field or the same register.

\section{Appendix 2}

Some hypothesis for the teachers

We have list general hypothesis, concerning only the cognitive point of view, that may help the teachers when choosing their scenario and their classroom's management but that each of them has to adapt to the precise content he wants to teach, to his students, etc.

1) In relation to the contents' choices 
Apart from conceiving an appropriate scenario (cf. page 2), more local decisions may occur.

a) To introduce a new notion

Depending on the very type of the concept (cf. supra), one can or cannot find a "good" problem making the students easier to apprehend the meaning of the concept (cf. pages 11-12, 17-18).

b) To work on a notion

Solve some SIT seems indispensable. But conceptualizing depends also in particular on the variety of tasks that the teacher suggests solving during the lesson. If there are only simple and isolated tasks (SIT), one may guess that the students will lack some tools to adapt their knowledge (cf. pages 14-15).

The order of the tasks is another "variable" on which the teacher may play.

Working on complex problems, involving not only one notion in one field, may also increase the level of available knowledge so as mixing new and old knowledge.

c) The obligation of writing

It allows an important and useful students' work on rigor and precision.

For instance writing completely a proof let students realize that they had not consider particular cases, that their notations were incomplete, etc...

It helps to understand precisely what is involved in reasoning.

d) The presentations of the lesson

In every case, the moments of teacher's presentation of knowledge are indispensable, to define and formalize what the students have to know. And it is yet more important when students have work by themselves before, so that they need to be informed of the corresponding knowledge.

Of course if there is nothing else during the classroom that this kind of teacher's presentation, one may guess that many students will switch off.

2) In relation to the class management's choices

a) Various tasks with time to solve them

The general idea is, in relation with the corresponding tasks, to choose an appropriate management. For instance when the tasks are complex, the idea is to associate a management that let students work alone, without the teacher during some time, so that he can afterwards lean on the students' actual work to make them go up.

b) Autonomous work in class (or in small groups)

It is important to give students occasions to work by themselves, to discuss between them, eventually to work in small groups, and to give individual appropriate assistance when there is a need of it. But it is also important to detect what the students have done when working alone and to make the most of it.

c) Habits 
It is when repeating sometimes a way of unusual work that the students may benefit of it.

d) Home work

It is important to give homework that all the students may realize, to improve them.

e) Appropriate assistance (pages 21-22)

There are many types of assistance and it is important to choose the moment to deliver them - before the work on a task, during it or after it. They may be general or particular, direct or not, they may take the shape of questions, or explanations... The important thing is to adapt the assistance to the students' question and knowledge.

\section{NOTES}

${ }^{1}$ This corresponds to what Rogalski refers to under the term "open dynamic environment management" in the previous chapter

${ }^{2}$ See previous section

${ }^{3}$ Knowledge presentation phase

${ }^{4}$ The importance and variability of the relationship with knowledge, in terms of the student's sociocultural origin, and the potential weight of emotional factors tied to the parents' level of schooling (Charlot, et al., 1992 ; Bautier, 2006 ; Bautier et Rochex, 1998).

${ }^{5}$ The Zone of Educational Priority includes institutions attended primarily by underprivileged students.

${ }^{6}$ For the most part. In some studies, the teacher's activity is examined in terms of its effects on the teacher (cf. regulation loop).

${ }^{7}$ In no case do we consider the (nonetheless important) domain of the unconscious.

${ }^{8}$ The idea of "availability" of these aspects is our way of translating the characteristic invariance of acquisitions under Vergnaud's models.

${ }^{9}$ Less and less, if we look at the current direction of French curricula.

${ }^{10}$ Despite what might be implied by the word "level."

${ }^{11}$ In-class lessons and/or textbook lessons.

${ }^{12}$ In certain studies, we speak of complex tasks.

${ }^{13}$ This is less frequent in general outside of university level classes.

14 "Concept" should be understood broadly, and includes some important theorems that are the object of a chapter.

${ }^{15}$ As defined by Brousseau (1990): The respective expectations of teachers and students.

${ }^{16}$ A chronology of class periods is established, based on the a priori task analysis and the effective unfolding of in-class events.

${ }^{17}$ Studies on the environment should be inserted here.

${ }^{18} \mathrm{Cf}$. Vygotsky, tied to his social analysis of knowledge under which "the collective appropriation may precede individual appropriation” (Vygotsky, thought and language).

${ }^{19}$ On a étudié les formats en présence, comme 'questions-réponses-reprises' ou la succession des prises de parole, en dégageant des types d'interaction, par-delà les contenus. Peut-être quelques exemples en note?

${ }^{20}$ Proximal development zone [à expliciter : il s 'agit de connaissances presque acquises que l'élève peut utiliser avec l'aide d'un adulte ou d'un pair, cf. Rogalski ?]

${ }^{21}$ In the sense of the double regulation schematic from part 0 .

${ }^{22}$ We find again here the idea of assistance with a constructive function.

${ }^{23}$ Establishments for educating future primary school teachers. 
ALINE ROBERT, CHRISTOPHE HACHE

${ }^{24}$ Professional establishments for training future teachers.

${ }^{25}$ Short for "didactic and ergonomic double approach" for the analyses of teaching practices (Robert \& Rogalski, 2002).

${ }^{26}$ In our initial findings, we described the way activities are set into motion, as well as the maintenance of students in the activity, called the student investment or more broadly, "keeping the class going."

${ }^{27}$ With a restriction: They place themselves within the approach they are helping to legitimize.

${ }^{28}$ This result comes from one of the OPEN (Observation of educational and teaching practices, 2008) subgroups concerning "practice organizers." In this subgroup, researchers in professional and other forms of didactics worked together with sociologists. Researchers were invited to respond to the question "For you, how should the term 'practice organizer' be defined?"

${ }^{29}$ What ergonomists call "discretionary tasks."

${ }^{30}$ There is a very important discussion here on the information supplied by evaluations.

${ }^{31}$ The levels of organization introduced in the appendix, which also take into account the flow of activities and the timeframe of the action, should also be distinguished.

${ }^{32}$ In previous studies, we have used the terms "lines of action" and "singularization" in discussing the macro and local levels.

${ }^{33}$ Vandebrouck (2002).

${ }^{34}$ Subdivisions on the order of several minutes, punctuated by activities organized for students.

${ }^{35}$ We have established these assessments of 9th and 10th grade (predominantly algebra) class periods after careful study. The problems given to students were not exercises of immediate application, but were introduced just before or after a lesson, and did not stray far from the lesson.

${ }^{36}$ This is, however, one of the strongest assessments made of the knowledge CAPES students developed at the university.

${ }^{37}$ Cf. Robert, Roditi, \& Grugeon [revue (2007), Diversité des offres de formation et travail du formateur d'enseignants de mathématiques du secondaire, Petit $x, \mathrm{n}^{\circ} 74$, p. 60-90].me donner plutôt une référence

${ }^{38}$ A theory that does not seem to us to be contradictory, but complementary to what we do, but which we would not be able to summarize briefly.

${ }^{39}$ Also impossible to summarize briefly

${ }^{40}$ In short, there should be no surprises.

\section{Aline Robert}

Christophe Hache

Laboratoire de Didactique André Revuz,

Université Paris Diderot 